\title{
Isomorphic tetravalent cyclic Haar graphs
}

\author{
Hiroki Koike \\ University of Primorska, UP IAM, Muzejski trg 2, SI6000 Koper, Slovenia \\ István Kovács * \\ University of Primorska, UP IAM and UP FAMNIT \\ Muzejski trg 2, SI6000 Koper, Slovenia
}

Received 31 January 2012, accepted 20 April 2013, published online 26 April 2013

\begin{abstract}
Let $S$ be a subset of the cyclic group $\mathbb{Z}_{n}$. The cyclic Haar graph $H\left(\mathbb{Z}_{n}, S\right)$ is the bipartite graph with color classes $\mathbb{Z}_{n}^{+}$and $\mathbb{Z}_{n}^{-}$, and edges $\left\{x^{+}, y^{-}\right\}$, where $x, y \in \mathbb{Z}_{n}$ and $y-x \in S$. In this paper we give sufficient and necessary conditions for the isomorphism of two connected cyclic Haar graphs of valency 4.
\end{abstract}

Keywords: Graph isomorphism, cyclic Haar graph, 4-BCI-group.

Math. Subj. Class.: 20B25, 05C25, 05C60

\section{Introduction}

Let $S$ be a subset of a finite group $G$. The Haar graph $H(G, S)$ is the bipartite graph with color classes identified with $G$ and written as $G^{+}$and $G^{-}$, and the edges are $\left\{x^{+}, y^{-}\right\}$, where $x, y \in G$ and $y x^{-1} \in S$. Haar graphs were introduced for abelian groups by Hladnik, Marušič and Pisanski [6], and were redefined under the name bi-Cayley graphs in [17]. A Haar graph $H(G, S)$ is called cyclic if $G$ is a cyclic group. In this paper we consider the problem of giving sufficient and necessary conditions for the isomorphism of two cyclic Haar graphs. This is a natural continuation of the isomorphism problem of circulant digraphs which has been solved by Muzychuk [12]. It appears in the context of circulant matrices under the name bipartite Ádám problem [16], and also in the context of cyclic configurations $[2,6]$.

The symbol $\mathbb{Z}_{n}$ denotes the additive group of the ring $\mathbb{Z} / n \mathbb{Z}$ of residue classes modulo $n$, and $\mathbb{Z}_{n}^{*}$ denotes the multiplicative group of units in $\mathbb{Z} / n \mathbb{Z}$. Two Haar graphs $H\left(\mathbb{Z}_{n}, S\right)$ and $H\left(\mathbb{Z}_{n}, T\right)$ are called affinely equivalent, written as $H\left(\mathbb{Z}_{n}, S\right) \cong_{\text {aff }} H\left(\mathbb{Z}_{n}, T\right)$, if $S$

\footnotetext{
${ }^{*}$ Corresponding author.

E-mail addresses: hiroki.koike@upr.si (Hiroki Koike), istvan.kovacs@upr.si (István Kovács)
} 
can be mapped to $T$ by an affine transformation, i.e., $a S+b=T$ for some $a \in \mathbb{Z}_{n}^{*}$ and $b \in \mathbb{Z}_{n}$. It is an easy exercise to show that two affinely equivalent cyclic Haar graphs are isomorphic as usual graphs. The converse implication is not true in general, and this makes the following definition interesting (see [17]): we say that a subset $S \subseteq \mathbb{Z}_{n}$ is a $B C I$ subset if for each $T \subseteq G, H\left(\mathbb{Z}_{n}, S\right) \cong H\left(\mathbb{Z}_{n}, T\right)$ if and only if $H\left(\mathbb{Z}_{n}, S\right) \cong$ aff $H\left(\mathbb{Z}_{n}, T\right)$. Wiedemann and Zieve proved in [16, Theorem 1.1] that any subset $S$ of $\mathbb{Z}_{n}$ is a BCI-subset if $|S| \leq 3$ (a special case was proved earlier in [3]). However, this is not true if $|S| \geq 4$ (see $[6,16])$, hence the first nontrivial case of the isomorphism problem occurs when $|S|=4$. In this paper we settle this case by proving the following theorem:

Theorem 1.1. Two connected Haar graphs $H\left(\mathbb{Z}_{n}, S\right)$ and $H\left(\mathbb{Z}_{n}, T\right)$ with $|S|=|T|=4$ are isomorphic if and only if there exist $a_{1}, a_{2} \in \mathbb{Z}_{n}^{*}$ and $b_{1}, b_{2} \in \mathbb{Z}_{n}$ such that

(1) $a_{1} S+b_{1}=T$; or

(2) $a_{1} S+b_{1}=\{0, u, v, v+m\}$ and $a_{2} T+b_{2}=\{0, u+m, v, v+m\}$, where $n=2 m$, $\mathbb{Z}_{n}=\langle u, v\rangle, 2|u, 2 u| m$ and $u / 2 \not \equiv v+m /(2 u)(\bmod m / u)$.

Remark 1.2. A group $G$ is called an $m$-BCI-group if every subset $S$ of $G$ with $|S| \leq m$ is a BCI-subset (see [7, 17]). In this context [16, Theorem 1.1] can be rephrased as $\mathbb{Z}_{n}$ is a 3 -BCI-group for any number $n$; and Theorem 1.1 says that $\mathbb{Z}_{n}$ is not a 4 -BCI-group if and only if $n$ is divisible by 8 . This refines [16, Theorem 7.2] in which it is proved that, if $\mathbb{Z}_{n}$ contains a non-BCI-subset of size $k, k \in\{4,5\}$, then $n$ has a prime divisor less or equal to $2 k(k-1)$.

Our approach towards Theorem 1.1 is group theoretical, we adopt the ideas of [1, 11]. In short terms the initial problem is transformed to a problem about the automorphism group of the graphs in question. Theorem 1.1 is proven in two steps: first it is settled for graphs $H\left(\mathbb{Z}_{n}, S\right)$ with $S$ satisfying additional conditions (see Theorem 3.1); then it is shown that, if $S$ is not a BCI-subset, then it is affinely equivalent to a set satisfying the conditions of Theorem 3.1 (see Theorem 4.1).

We conclude the introduction with the following modification of Theorem 1.1:

Theorem 1.3. Two connected Haar graphs $H\left(\mathbb{Z}_{n}, S\right)$ and $H\left(\mathbb{Z}_{n}, T\right)$ with $|S|=|T|=4$ are isomorphic if and only if there exist $a_{1}, a_{2} \in \mathbb{Z}_{n}^{*}$ and $b_{1}, b_{2} \in \mathbb{Z}_{n}$ such that

(1) $a_{1} S+b_{1}=T$; or

(2) $a_{1} S+b_{1}=\{0, u, v, v+m\}$ and $a_{2} T+b_{2}=\{0, u+m, v, v+m\}$, where $n=2 m$, $\mathbb{Z}_{n}=\langle u, v\rangle, 2|u, 2 u| m$.

Proof. In view of Theorem 1.1 it is sufficient to prove that $H\left(\mathbb{Z}_{n}, S\right) \cong H\left(\mathbb{Z}_{n}, T\right)$ if

$$
a_{1} S+b_{1}=\{0, u, v, v+m\} \text { and } a_{2} T+b_{2}=\{0, u+m, v, v+m\}
$$

where $n=2 m, \mathbb{Z}_{n}=\langle u, v\rangle, 2|u, 2 u| m$ and $u / 2 \equiv v+m /(2 u)(\bmod m / u)$. In fact, we are going to show below that there exist $a \in \mathbb{Z}_{n}^{*}$ and $b \in \mathbb{Z}_{n}$ such that

$$
a \cdot\{0, u, v, v+m\}+b=\{0, u+m, v, v+m\} .
$$

Then $\left(a_{2}^{-1} a a_{1}\right) \cdot S+a_{2}^{-1}\left(a b_{1}+b-b_{2}\right)=T$, and so $H\left(\mathbb{Z}_{n}, S\right) \cong H\left(\mathbb{Z}_{n}, T\right)$. 
Let us consider the following system of congruences:

$$
u x \equiv-u+m(\bmod n) \text { and } v x \equiv-u+v(\bmod n) .
$$

By the first congruence, using also that $2 u \mid m, x$ may be written in the form $x=$ $(n / u) y-1+m / u$. Plugging this in the second one, we obtain $(v n / u) y \equiv 2 v-u-$ $v m / u(\bmod n)$, which has an integer solution in $y$ exactly when $\operatorname{gcd}(v n / u, n) \mid(2 v-u-$ $v m / u)$. Then $\operatorname{gcd}(v n / u, n)=n / u \operatorname{gcd}(u, v)$, and since $\mathbb{Z}_{n}=\langle u, v\rangle, n / u$ and $\operatorname{gcd}(u, v)$ are coprime. Since $\operatorname{gcd}(u, v)$ is clearly a divisor of $2 v-u-v m / u$, a solution in $y$ exists if and only if $n / u \mid(2 v-u-v m / u)$, i.e., $u \equiv 2 v-v m / u(\bmod 2 m / u)($ recall that $n=2 m)$. On the other hand, one of the initial assumptions is $u / 2 \equiv v+m /(2 u)(\bmod m / u)$, and so $u \equiv 2 v+m / u(\bmod 2 m / u)$. We conclude that $(1.1)$ has an integer solution if $-v m / u \equiv$ $m / u(\bmod 2 m / u)$. Now, the latter congruence holds because of the conditions $2 \mid u$, $2 \mid n$, and $\mathbb{Z}_{n}=\langle u, v\rangle$. Let $a$ be a solution of (1.1). It follows from the above argument that $\operatorname{gcd}(a, m / u)=1$. Notice that, since $2 u \mid m, 2 \nmid a$. Let $d=\operatorname{gcd}(a, u)$. By (1.1), $a v \equiv-u+v(\bmod n)$, implying that $d \mid v$, and so $d=1$. We see that $\operatorname{gcd}(a, 2 m)=1$, i.e., $a \in \mathbb{Z}_{n}^{*}$. Choosing $b=u+m$, we finally get by (1.1), $a \cdot 0+b=u+m, a u+b=0$, $a v+b=v+m$, and $a(v+m)+b=v$, as required.

Theorem 1.3 becomes especially interesting when we compare it with the solution of the isomorphism problem of trivalent circulant digraphs. In fact, the same conditions can be derived from Muzychuk's general algorithm presented in [12]: two connected Cayley digraphs $\operatorname{Cay}\left(\mathbb{Z}_{n}, S\right)$ and $\operatorname{Cay}\left(\mathbb{Z}_{n}, T\right)$ with $|S|=|T|=3$ are isomorphic if and only if there exist $a_{1}, a_{2} \in \mathbb{Z}_{n}^{*}$ such that

- $a_{1} S=T$; or

- $a_{1} S=\{u, v, v+m\}$ and $a_{2} T=\{u+m, v, v+m\}$, where $n=2 m, \mathbb{Z}_{n}=\langle u, v\rangle$, $2 \mid u$, and $2 u \mid m$.

The natural question arises whether this phenomenon holds also for graphs of larger valencies.

\section{A Babai type theorem}

In this paper every group, graph and digraph is finite. For a (di)graph $\Gamma$, the symbols $V(\Gamma)$, $E(\Gamma)$ and $\operatorname{Aut}(\Gamma)$ denote the set of its vertices, (directed) edges and the full group of its automorphisms, respectively. Regarding terminology and notation in permutation group theory we follow [5].

Let $S$ be a subset of a group $G$. The Cayley digraph $\operatorname{Cay}(G, S)$ is the digraph with vertex set $G$, and its directed edges are $(x, y)$, where $x, y \in G$ and $y x^{-1} \in S$. Two digraphs $\operatorname{Cay}(G, S)$ and $\operatorname{Cay}(G, T)$ are called Cayley isomorphic, written as $\operatorname{Cay}(G, S) \cong_{\text {cay }}$ $\operatorname{Cay}(G, T)$, if $T=S^{\varphi}$ for some group automorphism $\varphi \in \operatorname{Aut}(G)$. It is clear that such an automorphism induces an isomorphism between $\operatorname{Cay}(G, S)$ and $\operatorname{Cay}(G, T)$, and thus Cayley isomorphic digraphs are isomorphic as usual digraphs. It is also well-known that the converse implication is not true, and this makes sense for the following definition (see [1]): a subset $S \subseteq G$ is a $C I$-subset if for each $T \subseteq G$, $\operatorname{Cay}(G, S) \cong \operatorname{Cay}(G, T)$ if and only if $\operatorname{Cay}(G, S) \cong{ }_{\text {cay }} \operatorname{Cay}(G, T)$. The following equivalence was proved by Babai [1, 3.1 Lemma].

Theorem 2.1. The following are equivalent for every Cayley digraph $\operatorname{Cay}(G, S)$. 
(1) S is a CI-subset.

(2) Every two regular subgroups of $\operatorname{Aut}(\operatorname{Cay}(G, S))$ isomorphic to $G$ are conjugate in $\operatorname{Aut}(\operatorname{Cay}(G, S))$.

Theorem 2.1 essentially says that the CI-property of a given subset $S$ depends entirely on the automorphism group $\operatorname{Aut}(\operatorname{Cay}(G, S))$. In this section we prove analogous results for cyclic Haar graphs.

Let $V=V\left(H\left(\mathbb{Z}_{n}, S\right)\right)$ be the vertex set of the Haar Graph $H\left(\mathbb{Z}_{n}, S\right)$. Throughout this paper $c$ and $d$ denote the permutations of $\mathrm{V}$ defined by

$$
c: x^{\varepsilon} \mapsto(x+1)^{\varepsilon} \text { and } d: x^{\varepsilon} \mapsto \begin{cases}(n-x)^{-} & \text {if } \varepsilon=+, \\ (n-x)^{+} & \text {if } \varepsilon=-,\end{cases}
$$

where $x \in \mathbb{Z}_{n}$ and $\varepsilon \in\{+,-\}$. It follows immediately that both $c$ and $d$ are automorphisms of any Haar graph $H\left(\mathbb{Z}_{n}, S\right)$. Denote by $C$ the group generated by $c$, and by $D$ the group generated by $c$ and $d$. The group $D$ acts regularly on $V$, and $D$ is isomorphic to $D_{2 n}$. Thus $H\left(\mathbb{Z}_{n}, S\right)$ is isomorphic to a Cayley graph over $D$, and so Theorem 2.1 can be applied. The following corollary is obtained.

Corollary 2.2. The implication $(1) \Leftarrow(2)$ holds for every Haar graph $H\left(\mathbb{Z}_{n}, S\right)$.

(1) S is a BCI-subset.

(2) Every two regular subgroups of $\operatorname{Aut}\left(H\left(\mathbb{Z}_{n}, S\right)\right)$ isomorphic to $D$ are conjugate in $\operatorname{Aut}\left(H\left(\mathbb{Z}_{n}, S\right)\right)$.

However, we do not have equivalence in Corollary 2.2 as it is shown in the following example.

Example 2.3. Let $\Gamma=H\left(\mathbb{Z}_{10},\{0,1,3,4\}\right)$. Using the computer package MAGMA [4] we compute that $\Gamma$ is edge-transitive and its automorphism group $\operatorname{Aut}(\Gamma) \cong D_{20} \rtimes \mathbb{Z}_{4}$. Furthermore, $\operatorname{Aut}(\Gamma)$ contains a regular subgroup $X$ which is isomorphic to $D_{20}$ but $X \neq$ $D_{20}$, hence (2) in Corollary 2.2 does not hold.

On the other hand, we find that for every subset $T \subseteq \mathbb{Z}_{10}$ with $0 \in T$ and $|T|=4$, the corresponding Haar graph $H\left(\mathbb{Z}_{10}, T\right) \cong \Gamma$ exactly when $H\left(\mathbb{Z}_{10}, T\right) \cong$ aff $\Gamma$. Thus $\{0,1,3,4\}$ is a BCI-subset, so (1) in Corollary 2.2 holds.

Example 2.3 shows that the isomorphism problem of cyclic Haar graphs is not a particular case of the isomorphism problem of Cayley graphs over dihedral groups. We remark that the latter problem is still unsolved, for partial solutions, see [1, 13, 14]. Nonetheless, the idea of Babai works well if instead of the regular subgroup $D$ we consider its index 2 cyclic subgroup $C$.

We say that a permutation group $G \leq \operatorname{Sym}\left(\mathbb{Z}_{n}^{+} \cup \mathbb{Z}_{n}^{-}\right)$is bicyclic if $G$ is a cyclic group which has two orbits: $\mathbb{Z}_{n}^{+}$and $\mathbb{Z}_{n}^{-}$. By a bicyclic group of a Haar graph $\Gamma=H\left(\mathbb{Z}_{n}, S\right)$ we simply mean a bicyclic subgroup $X \leq \operatorname{Aut}(\Gamma)$. Obviously, $C$ is a bicyclic group of any cyclic Haar graph, and being so it will be referred to as the canonical bicyclic group.

Let $\operatorname{Iso}(\Gamma)$ denote the set of all isomorphisms from $\Gamma$ to any other Haar graph $H\left(\mathbb{Z}_{n}\right.$, $T)$, i.e.,

$$
\operatorname{Iso}(\Gamma)=\left\{f \in \operatorname{Sym}(V): \Gamma^{f}=H\left(\mathbb{Z}_{n}, T\right) \text { for some } T \subseteq \mathbb{Z}_{n}\right\} .
$$


And let $\mathcal{C}_{\text {iso }}(\Gamma)$ denote the isomorphism class of cyclic Haar graphs which contains $\Gamma$, i.e., $\mathcal{C}_{\text {iso }}(\Gamma)=\left\{\Gamma^{f}: f \in \operatorname{Iso}(\Gamma)\right\}$.

Lemma 2.4. Let $\Gamma=H\left(\mathbb{Z}_{n}, S\right)$ be a connected Haar graph and $f$ be in $\operatorname{Sym}(V)$. Then $f \in \operatorname{Iso}(\Gamma)$ if and only if $f C f^{-1}$ is a bicyclic group of $\Gamma$.

Proof. Let $f \in \operatorname{Iso}(\Gamma)$. Then $f C f^{-1} \leq \operatorname{Aut}(\Gamma)$. Clearly, $f C f^{-1}$ is a cyclic group. Since the sets $\mathbb{Z}_{n}^{+}$and $\mathbb{Z}_{n}^{-}$are the color classes of the connected bipartite graph $\Gamma, f$ preserves these color classes, implying that $\operatorname{Orb}\left(f C f^{-1}, V\right)=\left\{\mathbb{Z}_{n}^{+}, \mathbb{Z}_{n}^{-}\right\}$. The group $f C f^{-1}$ is a bicyclic group of $\Gamma$.

Conversely, suppose that $f C f^{-1}$ is a bicyclic group of $\Gamma$. Then $C=f^{-1}\left(f C f^{-1}\right) f \leq$ $\operatorname{Aut}\left(\Gamma^{f}\right)$. Because that $\operatorname{Orb}\left(f C f^{-1}, V\right)=\left\{\mathbb{Z}_{n}^{+}, \mathbb{Z}_{n}^{-}\right\}$, the graph $\Gamma^{f}$ is connected and bipartite with color classes $\mathbb{Z}_{n}^{+}$and $\mathbb{Z}_{n}^{-}$. We conclude that $\Gamma^{f}=H\left(\mathbb{Z}_{n}, T\right)$ for some $T \subseteq \mathbb{Z}_{n}$, so $f \in \operatorname{Iso}(\Gamma)$. The lemma is proved.

Lemma 2.4 shows that the normalizer $N_{\operatorname{Sym}(V)}(C) \subseteq \operatorname{Iso}\left(H\left(\mathbb{Z}_{n}, S\right)\right)$. The group $N_{\mathrm{Sym}(V)}(C)$ is known to consist of the following permutations:

$$
\varphi_{r, s, t}: x^{\varepsilon} \mapsto\left\{\begin{array}{ll}
(r x+s)^{+} & \text {if } \varepsilon=+, \\
(r x+t)^{-} & \text {if } \varepsilon=-,
\end{array} \psi_{r, s, t}: x^{\varepsilon} \mapsto \begin{cases}(r x+s)^{-} & \text {if } \varepsilon=+, \\
(r x+t)^{+} & \text {if } \varepsilon=-,\end{cases}\right.
$$

where $r \in \mathbb{Z}_{n}^{*}$ and $s, t \in \mathbb{Z}_{n}$. Note that, two Haar graphs $H\left(\mathbb{Z}_{n}, S\right)$ and $H\left(\mathbb{Z}_{n}, T\right)$ are from the same orbit under $N_{\text {Sym }(V)}(C)$ exactly when $H\left(\mathbb{Z}_{n}, S\right) \cong \cong_{\text {aff }} H\left(\mathbb{Z}_{n}, T\right)$. Let $\mathcal{C}_{\text {aff }}(\Gamma)$ denote the affine equivalence class of cyclic Haar graphs which contains the graph $\Gamma=H\left(\mathbb{Z}_{n}, S\right)$, i.e., $\mathcal{C}_{\text {aff }}(\Gamma)=\left\{\Gamma^{\varphi}: \varphi \in N_{\mathrm{Sym}(V)}(C)\right\}$. It is clear that the isomorphism class $\mathcal{C}_{\text {iso }}(\Gamma)$ splits into affine equivalence classes:

$$
\mathcal{C}_{\text {iso }}(\Gamma)=\mathcal{C}_{\text {aff }}\left(\Gamma_{1}\right) \dot{\cup} \cdots \dot{\cup} \mathcal{C}_{\text {aff }}\left(\Gamma_{k}\right)^{1}
$$

Our next goal is to describe the above decomposition with the aid of bicyclic groups. Let $X$ be a bicyclic group of a connected graph $\Gamma=H\left(\mathbb{Z}_{n}, S\right)$. Then $g^{-1} X g$ is also a bicyclic group for every $g \in \operatorname{Aut}(\Gamma)$, hence the full set of bicyclic groups of $\Gamma$ is the union of Aut $(\Gamma)$-conjugacy classes. We say that a subset $\Xi \subseteq \operatorname{Iso}(\Gamma)$ is a bicyclic base of $\Gamma$ if the subgroups $\xi C \xi^{-1}, \xi \in \Xi$, form a complete set of representatives of the corresponding conjugacy classes. Thus every bicyclic group $X$ can be expressed as

$$
X=g \xi C(g \xi)^{-1} \text { for a unique } \xi \in \Xi \text { and } g \in \operatorname{Aut}(\Gamma) .
$$

Remark 2.5. Our definition of a bicyclic base copies in a sense the definition of a cyclic base introduced by Muzychuk [11, Definition, page 591].

Theorem 2.6. Let $\Gamma=H\left(\mathbb{Z}_{n}, S\right)$ be a connected Haar graph with a bicyclic base $\Xi$. Then $\mathcal{C}_{\text {iso }}(\Gamma)=\bigcup_{\xi \in \Xi} \mathcal{C}_{\text {aff }}\left(\Gamma^{\xi}\right)$.

Proof. It follows immediately that,

$$
\mathcal{C}_{\text {iso }}(\Gamma) \supseteq \bigcup_{\xi \in \Xi} \mathcal{C}_{\text {aff }}\left(\Gamma^{\xi}\right)
$$

\footnotetext{
${ }^{1}$ Here we mean that $\mathcal{C}_{\text {iso }}(\Gamma)=\mathcal{C}_{\text {aff }}\left(\Gamma_{1}\right) \cup \cdots \cup \mathcal{C}_{\text {aff }}\left(\Gamma_{k}\right)$ and $\mathcal{C}_{\text {aff }}\left(\Gamma_{i}\right) \cap \mathcal{C}_{\text {aff }}\left(\Gamma_{j}\right)=\emptyset$ for every $i, j \in\{1, \ldots, k\}, i \neq j$.
} 
We prove that equality holds in (2.3). Pick $\Sigma \in \mathcal{C}_{\text {iso }}(\Gamma)$. Then $\Sigma=\Gamma^{f}$ for some $f \in$ Iso $(\Gamma)$. By Lemma 2.4, $f C f^{-1}$ is a bicyclic group of $\Gamma$, hence

$$
f C f^{-1}=g \xi C(g \xi)^{-1}, \xi \in \Xi, g \in \operatorname{Aut}(\Gamma) .
$$

Thus $f^{-1} g \xi=h$, where $h \in N_{\operatorname{Sym}(V)}(C)$. Then

$$
\Sigma=\Gamma^{f}=\Gamma^{g \xi h^{-1}}=\left(\Gamma^{\xi}\right)^{h^{-1}} .
$$

This shows that $\Sigma \in \mathcal{C}_{\text {aff }}\left(\Gamma^{\xi}\right)$, and so

$$
\mathcal{C}_{\mathrm{iso}}(\Gamma) \subseteq \bigcup_{\xi \in \Xi} \mathcal{C}_{\mathrm{aff}}\left(\Gamma^{\xi}\right)
$$

In view of (2.3) the two sides are equal.

Moreover, if $\mathcal{C}_{\text {aff }}\left(\Gamma^{\xi_{1}}\right) \cap \mathcal{C}_{\text {aff }}\left(\Gamma^{\xi_{2}}\right) \neq \emptyset$ for $\xi_{1}, \xi_{2} \in \Xi$, then $\Gamma^{\xi_{1}}=\Gamma^{\xi_{2} h}$ for some $h \in N_{\mathrm{Sym}(V)}(C)$. Hence $\xi_{2} h \xi_{1}^{-1}=g$ for some $g \in \operatorname{Aut}(\Gamma)$, and so

$$
\xi_{1} C \xi_{1}^{-1}=g^{-1} \xi_{2} h C h^{-1} \xi_{2}^{-1} g=g^{-1}\left(\xi_{2} C \xi_{2}^{-1}\right) g .
$$

The bicyclic subgroups $\xi_{1} C \xi_{1}^{-1}$ and $\xi_{2} C \xi_{2}^{-1}$ are conjugate in $\operatorname{Aut}(\Gamma)$, hence $\xi_{1}=\xi_{2}$ follows from the definition of the bicyclic base $\Xi$. We obtain that $\mathcal{C}_{\text {aff }}\left(\Gamma^{\xi_{1}}\right) \cap \mathcal{C}_{\text {aff }}\left(\Gamma^{\xi_{2}}\right)=\emptyset$ whenever $\xi_{1}, \xi_{2} \in \Xi, \xi_{1} \neq \xi_{2}$, and so $\mathcal{C}_{\text {iso }}(\Gamma)=\dot{\bigcup}_{\xi \in \Xi} \mathcal{C}_{\text {aff }}\left(\Gamma^{\xi}\right)$. The theorem is proved.

As a direct consequence of Theorem 2.6 we obtain the following corollary, analog of Theorem 2.1.

Corollary 2.7. The following are equivalent for every connected Haar graph $H\left(\mathbb{Z}_{n}, S\right)$.

(1) S is a BCI-subset.

(2) Any two bicyclic groups of $H\left(\mathbb{Z}_{n}, S\right)$ are conjugate in $\operatorname{Aut}\left(H\left(\mathbb{Z}_{n}, S\right)\right)$.

In our last proposition we connect the BCI-property with the CI-property. For $a^{\varepsilon} \in V$, in what follows $\operatorname{Aut}\left(H\left(\mathbb{Z}_{n}, S\right)\right)_{a^{\varepsilon}}$ denotes the vertex stabilizer of $a^{\varepsilon}$ in $\operatorname{Aut}\left(H\left(\mathbb{Z}_{n}, S\right)\right)$.

Proposition 2.8. Suppose that $\Gamma=H\left(\mathbb{Z}_{n}, S\right)$ is a connected Haar graph such that for some $a \in \mathbb{Z}_{n}, \operatorname{Aut}(\Gamma)_{0^{+}}=\operatorname{Aut}(\Gamma)_{a^{-}}$. Then the following are equivalent.

(1) S is a BCI-subset.

(2) $S-a=\{s-a: s \in S\}$ is a CI-subset.

Proof. For sake of simplicity we put $A=\operatorname{Aut}(\Gamma)$ and $G=\operatorname{Aut}(\Gamma)_{\left\{\mathbb{Z}_{n}^{+}\right\}}$, i.e., the setwise stabilizer of the color class $\mathbb{Z}_{n}^{+}$in Aut $(\Gamma)$. Obviously, $X \leq G$ for every bicyclic group $X$ of $\Gamma$. Since $A=G \rtimes\langle d\rangle$ and $d$ normalizes $C$, it follows that the conjugacy class of subgroups of $A$ containing $C$ is equal to the conjugacy class of subgroups of $G$ containing $C$. Using this and Theorem 2.6, we obtain that $S$ is a BCI-subset if and only if every bicyclic group is conjugate to $C$ in $G$. 
Let $W=\left\{0^{+}, a^{-}\right\}$and consider the setwise stabilizer $A_{\{W\}}$. Since $A_{0^{+}}=A_{a^{-}}$, $A_{0^{+}} \leq A_{\{W\}}$. By [5, Theorem 1.5A], the orbit of $0^{+}$under $A_{\{W\}}$ is a block of imprimitivity (for short a block) for $A$. Denote this block by $\Delta$ and the induced system of blocks by $\delta$ (i.e., $\delta=\left\{\Delta^{g}: g \in G\right\}$ ). Consider the element $g=d c^{a}$ from $D$. We see that $g$ switches $0^{+}$and $a^{-}$, hence $A_{\{W\}}=A_{0^{+}}\langle g\rangle$. Therefore, $\Delta=\left(0^{+}\right)^{A_{\{W\}}}=\left(0^{+}\right)^{A_{0^{+}}}\langle g\rangle=$ $\left(0^{+}\right)^{\langle g\rangle}=W$, and so

$$
\delta=\left\{\left\{x^{+},(x+a)^{-}\right\}: x \in \mathbb{Z}_{n}\right\} .
$$

Define the mapping $\varphi: \delta \rightarrow \mathbb{Z}_{n}$ by $\varphi:\left\{x^{+},(x+a)^{-}\right\} \mapsto x, x \in \mathbb{Z}_{n}$. Now, an action of $A$ on $\mathbb{Z}_{n}$ can be defined by letting $g \in A$ act as

$$
x^{g}=x^{\varphi^{-1} g \varphi}, x \in \mathbb{Z}_{n}
$$

For $g \in A$ we write $\bar{g}$ for the image of $g$ under the corresponding permutation representation, and for a subgroup $X \leq A$ we let $\bar{X}=\{\bar{x}: x \in X\}$. In this action of $A$ the subgroup $G<A$ is faithful. Also notice that, a subgroup $X \leq G$ is a bicyclic group of $\Gamma$ if and only if $\bar{X}$ is a regular cyclic subgroup of $\bar{G}$. In particular, for the canonical bicyclic group $C$, $\bar{C}=\left(\mathbb{Z}_{n}\right)_{\text {right }}$, where $\left(\mathbb{Z}_{n}\right)_{\text {right }}$ denotes the group generated by the affine transformation $x \mapsto x+1, x \in \mathbb{Z}_{n}$.

Pick $g \in G$ and $(x, x+s-a) \in \mathbb{Z}_{n} \times \mathbb{Z}_{n}$, where $s \in S$. Then $g$ maps the directed edge $\left(x^{+},(x+s)^{-}\right)$to a directed edge $\left(y^{+},(y+q)^{-}\right)$for some $y \in \mathbb{Z}_{n}$ and $q \in S$. Since $\delta$ is a system of blocks for $G, g$ maps $(x+s-a)^{+}$to $(y+q-a)^{+}$, and so $\bar{g}$ maps the pair $(x, x+s-a)$ to the pair $(y, y+q-a)$. We have just proved that $\bar{g}$ leaves the set $\left\{(x, x+s-a): x \in \mathbb{Z}_{n}, s \in S\right\}$ setwise fixed. As the latter set is the set of all directed edges of the digraph $\operatorname{Cay}\left(\mathbb{Z}_{n}, S-a\right), \bar{G} \leq \operatorname{Aut}\left(\operatorname{Cay}\left(\mathbb{Z}_{n}, S-a\right)\right)$. For an automorphism $h$ of Cay $\left(\mathbb{Z}_{n}, S-a\right)$, define the permutation $g$ of $V$ by

$$
g: x^{\varepsilon} \mapsto\left\{\begin{array}{ll}
\left(x^{h}\right)^{+} & \text {if } \varepsilon=+, \\
\left((x-a)^{h}+a\right)^{-} & \text {if } \varepsilon=-,
\end{array} \quad x \in \mathbb{Z}_{n}, \varepsilon \in\{+,-\} .\right.
$$

The reader is invited to check that the above permutation $g$ is an automorphism of $\Gamma$. It is clear that $g \in G$ and $\bar{g}=h$; we conclude that $\bar{G}=\operatorname{Aut}\left(\operatorname{Cay}\left(\mathbb{Z}_{n}, S-a\right)\right)$.

Now, the proposition follows along the following equivalences:

(1) $\Longleftrightarrow \quad$ Every bicyclic group of $\Gamma$ is conjugate to $C$ in $G$

$\Longleftrightarrow \quad$ Every regular cyclic subgroup of $\bar{G}$ is conjugate to $\bar{C}$ in $\bar{G}$

$\Longleftrightarrow \quad(2)$

The last equivalence is Theorem 2.1 .

Remark 2.9. Let us remark that the equality $\operatorname{Aut}(\Gamma)_{0^{+}}=\operatorname{Aut}(\Gamma)_{a^{-}}$does not hold in general. For example, take $\Gamma$ as the incidence graph of the projective space $\operatorname{PG}(d, q)$ where $d \geq 2$ and $q$ is a prime power (i.e., $\Gamma$ is the bipartite graph whose color classes are identified by the set of points and the set of hyperplanes, respectively, and the edges are defined by the incidence relation of the space). It is well-known that $\operatorname{PG}(d, q)$ admits a cyclic group of automorphisms (called a Singer subgroup) acting regularly on both the points and the hyperplanes. This shows that $\Gamma$ is isomorphic to a cyclic Haar graph, and we may identify the set of points with $\mathbb{Z}_{n}^{+}$, and the set of hyperplanes with $\mathbb{Z}_{n}^{-}$, where 
$n=\left(q^{d}-1\right) /(q-1)$. The automorphism group $\operatorname{Aut}(\Gamma)=\operatorname{P\Gamma L}(d+1, q) \rtimes \mathbb{Z}_{2}$; and as $\operatorname{P\Gamma L}(d+1, q)$ acts inequivalently on the points and the hyperplanes, $\operatorname{Aut}(\Gamma)_{0^{+}}$cannot be equal to $\operatorname{Aut}(\Gamma)_{a^{-}}$for any $a \in \mathbb{Z}_{n}$.

\section{Haar graphs $H\left(\mathbb{Z}_{2 m},\{0, u, v, v+m\}\right)$}

In this section we prove Theorem 1.1 for Haar graphs $H\left(\mathbb{Z}_{n}, S\right)$ satisfying certain additional conditions.

Theorem 3.1. Let $n=2 m$ and $S=\{0, u, v, v+m\}$ such that

(a) $\mathbb{Z}_{n}=\langle u, v\rangle$

(b) $1<u<m, u \mid m$;

(c) $\operatorname{Aut}\left(H\left(\mathbb{Z}_{n}, S\right)\right)_{0^{+}}$leaves the set $\left\{0^{-}, u^{-}\right\}$setwise fixed.

Then $H\left(\mathbb{Z}_{n}, S\right) \cong H\left(\mathbb{Z}_{n}, T\right)$ if and only if there exist $a \in \mathbb{Z}_{n}^{*}$ and $b \in \mathbb{Z}_{n}$ such that

(1) $a T+b=S ;$ or

(2) $a T+b=\{0, u+m, v, v+m\}$, and $2|u, 2 u| m, u / 2 \not \equiv v+m /(2 u)(\bmod m / u)$.

By (b) of Theorem 3.1 we have $2 u \leq m$. We prove the extremal case, when $2 u=m$, separately. Notice that, in this case the conditions in (2) of Theorem 3.1 that $2|u, 2 u| m$ and $u / 2 \not \equiv v+m /(2 u)(\bmod m / u)$ can be replaced by one condition: $u \equiv 2(\bmod 4)$.

Lemma 3.2. Let $S$ be the set defined in Theorem 3.1. If $2 u=m$, then $H\left(\mathbb{Z}_{n}, S\right) \cong$ $H\left(\mathbb{Z}_{n}, T\right)$ if and only if there exist $a \in \mathbb{Z}_{n}^{*}$ and $b \in \mathbb{Z}_{n}$ such that

(1) $a T+b=S ;$ or

(2) $a T+b=\{0, u+m, v, v+m\}$ and $u \equiv 2(\bmod 4)$.

Proof. Let $d=\operatorname{gcd}(n, v)$. Because of $\langle u, v\rangle=\mathbb{Z}_{n}$ we have that $\operatorname{gcd}(u, v, n)=1$, i.e., $\operatorname{gcd}(n / 4, v)=1$, and this gives that $d \in\{1,2,4\}$. Note that, if $d \neq 1$, then necessarily $2 \nmid u$. Let us write $v=v_{1} d$, where $\operatorname{gcd}\left(v_{1}, n\right)=1$. Let $v_{1}^{-1}$ denote the inverse of $v_{1}$ in the group $\mathbb{Z}_{n}^{*}$. Then the following hold in $\mathbb{Z}_{n}$ (here we use that $u=n / 4$ ):

$$
v_{1}^{-1} v=d, v_{1}^{-1}(v+m)=d+m \text { and } v_{1}^{-1} u \in\{u, 3 u\} .
$$

We conclude that $S$ is affinely equivalent to one of the sets $S_{i}(d), i \in\{1,2\}$ and $d \in$ $\{1,2,4\}$, where

$$
S_{1}(d)=\{0, u, d, d+2 u\} \text { or } S_{2}(d)=\{0,3 u, d, d+2 u\} .
$$

The lemma follows from the following claims:

(i) $H\left(\mathbb{Z}_{n}, S_{1}(1)\right) \cong H\left(\mathbb{Z}_{n}, S_{2}(1)\right)$.

(ii) $H\left(\mathbb{Z}_{n}, S_{1}(1)\right) \cong$ aff $H\left(\mathbb{Z}_{n}, S_{1}(d)\right)$ for $d \in\{2,4\}$;

(iii) $H\left(\mathbb{Z}_{n}, S_{1}(d)\right) \cong$ aff $H\left(\mathbb{Z}_{n}, S_{2}(d)\right) \Longleftrightarrow d \in\{2,4\}$ or $(d=1$ and $u \not \equiv 2(\bmod 4))$; 

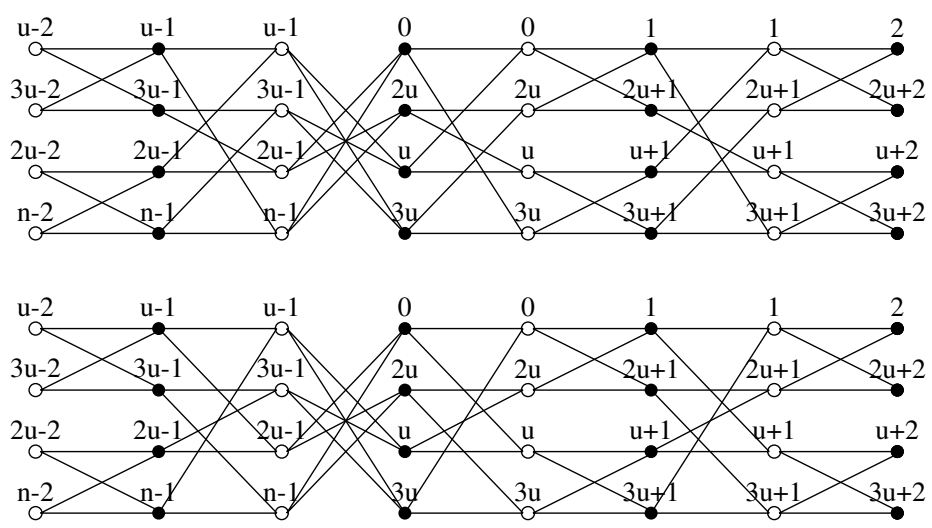

Figure 1: Haar graphs $H\left(\mathbb{Z}_{n}, S_{1}(1)\right)$ and $H\left(\mathbb{Z}_{n}, S_{2}(1)\right)$.

(i): Define the mapping $f: V \mapsto V$ by

$$
f: x^{\varepsilon} \mapsto \begin{cases}x^{\varepsilon} & \text { if } x \in\{0,1, \ldots u-1\} \cup\{2 u, \ldots 3 u-1\}, \\ (x+2 u)^{\varepsilon} & \text { otherwise. }\end{cases}
$$

We leave for the reader to verify that $f$ is in fact an isomorphism from $H\left(\mathbb{Z}_{n}, S_{1}(1)\right)$ to $H\left(\mathbb{Z}_{n}, S_{2}(1)\right)$ (compare the graphs in Figure 1; here the white vertices represent the color class $\mathbb{Z}_{n}^{+}$, while the black ones represent the color class $\mathbb{Z}_{n}^{-}$).

(ii): Since $d \in\{2,4\}, u$ is an odd number. For $d \in\{2,4\}$ define $r_{d} \in \mathbb{Z}_{n}^{*}$ as follows:

$$
r_{2}=\left\{\begin{array}{ll}
2+u & \text { if } u \equiv 1(\bmod 4), \\
2+3 u & \text { if } u \equiv 3(\bmod 4),
\end{array} \quad r_{4}= \begin{cases}4+u & \text { if } u \equiv 3(\bmod 4) \\
4+3 u & \text { if } u \equiv 1(\bmod 4)\end{cases}\right.
$$

It can be directly checked that $r_{d} S_{1}(1)+u=S_{1}(d)$, so $H\left(\mathbb{Z}_{n}, S_{1}(1)\right) \cong$ aff $H\left(\mathbb{Z}_{n}, S_{1}(d)\right)$ for $d \in\{2,4\}$.

(iii): If $u$ is odd, then $(2 u+1) S_{1}(d)=S_{2}(d)$, hence $H\left(\mathbb{Z}_{n}, S_{1}(d)\right) \cong$ aff $H\left(\mathbb{Z}_{n}, S_{2}(d)\right)$. Since $u$ is odd whenever $d \in\{2,4\}$, we are left with the case that $d=1$ and $u$ is even. If also $u \equiv 0(\bmod 4)$, then $(u+1) S_{1}(1)+3 u=S_{2}(1)$, and again $H\left(\mathbb{Z}_{n}, S_{1}(1)\right) \cong$ aff $H\left(\mathbb{Z}_{n}, S_{2}(1)\right)$.

Suppose that $d=1$ and $u \equiv 2(\bmod 4)$. We finish the proof by showing that in this case $H\left(\mathbb{Z}_{n}, S_{1}(1)\right) \approx_{\text {aff }} H\left(\mathbb{Z}_{n}, S_{2}(1)\right)$. Suppose that, there is an affine transformation $\psi: x \mapsto$ $r x+s, r \in \mathbb{Z}_{n}^{*}$ and $s \in \mathbb{Z}_{n}$, which maps the set $S_{1}(1)$ to $S_{1}(2)$. Then $1^{\psi}-(1+2 u)^{\psi}=2 u$ in $\mathbb{Z}_{n}$. This implies that $\{1,1+2 u\}^{\psi}=\{1,1+2 u\}$ and $\{0, u\}^{\psi}=\{0,3 u\}$, and hence

$$
r+s \in\{1,1+2 u\} \text { and } r\{0, u\}+s=\{0,3 u\} .
$$

A direct analysis shows that the above equations cannot hold if $u \equiv 2(\bmod 4)$. Thus $H\left(\mathbb{Z}_{n}, S_{1}(1)\right) \varlimsup_{\text {aff }} H\left(\mathbb{Z}_{n}, S_{2}(1)\right)$, this completes the proof of (iii).

Now, we turn to the case when $2 u \neq m$. Recall that the canonical bicyclic group $C$ is generated by the permutation $c$ defined in (1). For a divisor $\ell \mid n, C^{\ell}$ will denote the 


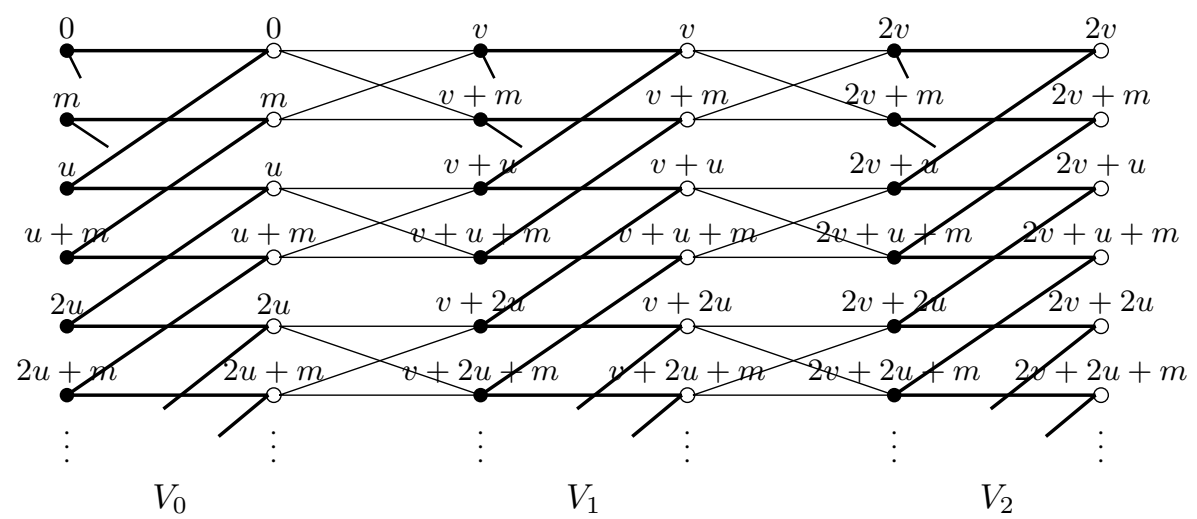

Figure 2: The Haar graph $H\left(\mathbb{Z}_{n}, S\right)$

subgroup of $C$ generated by $c^{\ell}$. It will be convenient to denote by $\delta_{\ell}$ the partition of $V$ into the orbits of $C^{\ell}$, i.e., $\delta_{\ell}=\operatorname{Orb}\left(C^{\ell}, V\right)$. Furthermore, we set $\eta_{n, \ell}$ for the homomorphism $\eta_{n, \ell}: \mathbb{Z}_{n} \rightarrow \mathbb{Z}_{\ell}$ defined by $\eta_{n, \ell}(1)=1$.

Observe that, if $\delta_{\ell}$ is, in addition, a system of blocks for the group $A=\operatorname{Aut}\left(H\left(\mathbb{Z}_{n}\right.\right.$, $S)$ ), then an action of $A$ can be defined on $H\left(\mathbb{Z}_{\ell}, \eta_{n, \ell}(S)\right)$ by letting $g \in A$ act as for $x \in \mathbb{Z}_{\ell}$ and for $\varepsilon, \varepsilon^{\prime} \in\{+,-\}$,

$$
\left(x^{\varepsilon}\right)^{g}=y^{\varepsilon^{\prime}} \Longleftrightarrow\left\{z^{\varepsilon}: z \in \eta_{n, \ell}^{-1}(x)\right\}^{g}=\left\{z^{\varepsilon^{\prime}}: z \in \eta_{n, \ell}^{-1}(y)\right\} .
$$

We denote by $A_{\left(\delta_{\ell}\right)}$ the corresponding kernel, and by $g^{\delta_{\ell}}$ the image of an element $g \in G$. Note that, if $X$ is a bicyclic group of $H\left(\mathbb{Z}_{n}, S\right)$, then $X^{\delta_{\ell}}=\left\{x^{\delta_{\ell}}: x \in X\right\}$ is a bicyclic group of $H\left(\mathbb{Z}_{\ell}, \eta_{n, \ell}(S)\right)$.

Let $S=\{0, u, v, v+m\}$ be the subset of $\mathbb{Z}_{n}$ defined in Theorem 3.1. Let $\delta$ be the partition of $V$ defined by

$$
\delta=\left\{X \cup X^{\psi_{1,0,0}}: X \in \operatorname{Orb}\left(C^{u}, V\right)\right\},
$$

where $\psi_{1,0,0}$ is defined in (2.2). We write $\delta=\left\{V_{0}, \ldots, V_{u-1}\right\}$, where

$$
V_{i}=\left\{(i v+j u)^{+},(i v+j u)^{-}: j \in\{0,1, \ldots,(n / u)-1\}\right\} .
$$

A part of $H\left(\mathbb{Z}_{n}, S\right)$ is drawn in Figure 2 using the partition $\delta$. White and black colors represent again the color classes $\mathbb{Z}_{n}^{+}$and $\mathbb{Z}_{n}^{-}$, respectively. For $i \in\{0,1, \ldots, u-1\}$, let $e_{i}$ be the involution of $V$ defined by

$$
e_{i}: x^{\varepsilon} \mapsto \begin{cases}(x+m)^{\varepsilon} & \text { if } x^{\varepsilon} \in V_{i}, \\ x^{\varepsilon} & \text { otherwise. }\end{cases}
$$

It is clear that each $e_{i} \in \operatorname{Aut}\left(H\left(\mathbb{Z}_{n}, S\right)\right)$, and also that $e_{i} e_{j}=e_{j} e_{i}$ for all $i, j \in\{0,1, \ldots$, $u-1\}$. Let $E=\left\langle e_{0}, e_{1}, \ldots, e_{u-1}\right\rangle$. Thus $E \leq \operatorname{Aut}\left(H\left(\mathbb{Z}_{n}, S\right)\right)$ and $E \cong \mathbb{Z}_{2}^{u}$. For a subset $I \subseteq\{0,1, \ldots, u-1\}$ let $e_{I}$ be the element in $E$ defined by $e_{I}=\prod_{i \in I} e_{i}$.

The following lemma about imprimitivity systems of blocks (systems of blocks for short) will be used throughout the paper. 
Lemma 3.3. Let $\Gamma=H\left(\mathbb{Z}_{n}, R\right)$ be a Haar graph and suppose that $R_{*} \subseteq R$ such that the point stabilizer $\operatorname{Aut}(\Gamma)_{0^{+}}$fixes setwise $R_{*}^{-}$, and let $d=\left|\left\langle R_{*}-R_{*}\right\rangle\right|$, where $R_{*}-R_{*}=$ $\left\{r_{1}-r_{2}: r_{1}, r_{2} \in R_{*}\right\}$. Then the partition $\pi$ of $V$ defined by

$$
\pi=\left\{X \cup X^{\psi_{1, r,-r}}: X \in \operatorname{Orb}\left(C^{n / d}, V\right)\right\}, \text { where } r \in R_{*},
$$

is a system of blocks for $\operatorname{Aut}(\Gamma){ }^{2}$

Proof. For short we set $A=\operatorname{Aut}(\Gamma)$. Since $R_{*}^{-}$is fixed setwise by $A_{0^{+}}$, we may write

$$
R_{*}=R_{1} \cup \cdots \cup R_{k},
$$

where $R_{i}^{-}$is an $A_{0^{+}}$-orbit for every $i \in\{1,2, \ldots, k\}$. Choose an arc $\left(0^{+}, r_{i}^{-}\right)$of $\Gamma$ where we fix an element $r_{i} \in R_{i}$ for every $i \in\{1, \ldots, k\}$. We claim that, the orbital graph of $A$ containing $\left(0^{+}, r_{i}^{-}\right)$is self-paired, and in fact it is equal to the Haar graph $H\left(\mathbb{Z}_{n}, R_{i}\right)$ (for a definition of an orbital graph, see [5]).

Define $\bar{A}$ as the color preserving subgroup of $A$. Then $A=\bar{A} \rtimes\left\langle\psi_{-1,0,0}\right\rangle$. Also, $\bar{A}=A_{0^{+}} C$, as $C$ is transitive on $\mathbb{Z}_{n}^{+}$. Then the orbit of the $\operatorname{arc}\left(0^{+}, r_{i}^{-}\right)$under $A$ is

$$
\begin{aligned}
& \left(0^{+}, r_{i}^{-}\right)^{A}=\left(0^{+}, r_{i}^{-}\right)^{A_{0^{+}} C\left\langle\psi_{-1,0,0}\right\rangle}=\left\{\left(0^{+}, r_{i}^{\prime-}\right): r_{i}^{\prime} \in R_{i}\right\}^{C\left\langle\psi_{-1,0,0}\right\rangle} \\
& =\left\{\left(j^{+},\left(j+r_{i}^{\prime}\right)^{-}\right): r_{i}^{\prime} \in R_{i}, j \in \mathbb{Z}_{n}\right\}^{\left\langle\psi_{-1,0,0}\right\rangle} \\
& =\left\{\left(j^{+},\left(j+r_{i}^{\prime}\right)^{-}\right): r_{i}^{\prime} \in R_{i}, j \in \mathbb{Z}_{n}\right\} \cup \\
& \left\{\left((-j)^{-},\left(-j-r_{i}^{\prime}\right)^{+}\right): r_{i}^{\prime} \in R_{i}, j \in \mathbb{Z}_{n}\right\}, \\
& =\left\{\left(j^{+},\left(j+r_{i}^{\prime}\right)^{-}\right): r_{i}^{\prime} \in R_{i}, j \in \mathbb{Z}_{n}\right\} \cup \\
& \left\{\left(\left(j+r_{i}^{\prime}\right)^{-}, j^{+}\right): r_{i}^{\prime} \in R_{i}, j \in \mathbb{Z}_{n}\right\},
\end{aligned}
$$

which is clearly equal to the set of arcs of $H\left(\mathbb{Z}_{n}, R_{i}\right)$. The claim is proved.

Since $H\left(\mathbb{Z}_{n}, R_{i}\right)$ is an orbital graph, $A \leq \operatorname{Aut}\left(H\left(\mathbb{Z}_{n}, R_{i}\right)\right)$. Combining this with $H\left(\mathbb{Z}_{n}, R_{*}\right)=\cup_{i=1}^{k} H\left(\mathbb{Z}_{n}, R_{i}\right)$, we have that $A \leq \operatorname{Aut}\left(H\left(\mathbb{Z}_{n}, R_{*}\right)\right.$. Let $\Sigma$ be the connected component of $H\left(\mathbb{Z}_{n}, R_{*}\right)$ which contains $0^{+}$. Obviously, the set $W$ of vertices contained in $\Sigma$ is a block for $A$. It is easy to verify that $W=X \cup X^{\psi_{1, r,-r}}$ where $X$ is the orbit of $0^{+}$under $C^{n / d}$. The lemma follows.

Lemma 3.4. Let $S$ be the set defined in Theorem 3.1. If $2 u \neq m$, then the stabilizer $\operatorname{Aut}\left(H\left(\mathbb{Z}_{n}, S\right)\right)_{0^{+}}$is given as follows.

(1) If $u \not \equiv 2 v(\bmod m / u)$, then $\operatorname{Aut}\left(H\left(\mathbb{Z}_{n}, S\right)\right)_{0^{+}}=E_{0^{+}}$.

(2) If $u \equiv 2 v(\bmod m / u)$, then $\operatorname{Aut}\left(H\left(\mathbb{Z}_{n}, S\right)\right)_{0^{+}}=E_{0^{+}} \times F$ for a subgroup $F \leq$ $\operatorname{Aut}\left(H\left(\mathbb{Z}_{n}, S\right)\right)_{0^{+}},|F|=2$.

Proof. For short we set $\Gamma=H\left(\mathbb{Z}_{n}, S\right)$ and $A=\operatorname{Aut}(\Gamma)$. Consider the partition $\delta$ defined in (3.2). Applying Lemma 3.3 with $R=S, R_{*}=\{0, u\}$ and $r=0$, we obtain that $\delta$ is a system of blocks for $A$. The quotient graph $\Gamma / \delta$ is a $u$-circuit if $u>2$ and a 2-path if $u=2$. Let $g \in A_{0^{+}}$. Then $g$ fixes the directed edge $\left(V_{0}, V_{1}\right)$ of $\Gamma / \delta$, hence it must fix all sets $V_{i}$. Thus $A_{0^{+}} \leq A_{(\delta)}$, where $A_{(\delta)}$ is the kernel of the action of $A$ on $\delta$.

Consider the action of $A_{0^{+}}$on $V_{0}$. The corresponding kernel is $A_{\left(V_{0}\right)}$, the pointwise stabilizer of $V_{0}$ in $A$, and the corresponding image is a subgroup of $\operatorname{Aut}\left(\Gamma\left[V_{0}\right]\right)$, where

\footnotetext{
${ }^{2}$ Notice that, $\pi$ does not depend of the choice of the element $r \in R_{*}$.
} 
$\Gamma\left[V_{0}\right]$ is the subgraph of $\Gamma$ induced by $V_{0}$. Using that $2 u \neq m$, we show next that $A_{\left(V_{0}\right)}=$ $E_{0^{+}}$. It is clear that $A_{\left(V_{0}\right)} \geq E_{0^{+}}$. We are going to prove that $A_{\left(V_{0}\right)} \leq E_{0^{+}}$also holds. Let $g \in A_{\left(V_{0}\right)}$. Then for a suitable element $e \in\left\langle e_{1}\right\rangle$, the product $g e$ fixes pointwise $V_{0}$ and fix the vertex $v^{-}$from block $V_{1}$ (see Figure 2). Thus ge acts on $V_{1}$ as the identity or the unique reflection of the circuit $\Gamma\left[V_{1}\right]$ that fixes $v^{-}$. If this action is not the identity, then $g e$ switches $v^{+}$and $(v+n-u)^{+}$, and so it must switch $(v+u)^{-}$and $(v+n-u)^{-}$. On the other hand, since $(v+u)^{-}$is connected to $u^{+} \in V_{0}$, it follows that $(v+u)^{-}$can only be mapped to $(v+u+m)^{-}$, and so $(v+n-u)^{-}=(v+u+m)^{-}$, contradicting that $2 u \neq m$. We conclude that $g e$ acts as the identity also on $V_{1}$. Continuing in this way, we find that $g e^{\prime}$ is the identity with a suitable choice of $e^{\prime} \in E_{0^{+}}$, hence $g=e^{\prime}$.

The equality $A_{\left(V_{0}\right)}=E_{0^{+}}$together with $\operatorname{Aut}\left(\Gamma\left[V_{0}\right]\right) \cong D_{4 u}$ imply that $\left|A_{0^{+}}: E_{0^{+}}\right| \leq$ 2. Moreover, $\left|A_{0^{+}}: E_{0^{+}}\right|=2$ holds exactly when $A_{0^{+}}$contains an involution $g$ for which $g: 0^{-} \leftrightarrow u^{-}$. In the latter case $A_{0^{+}}=E_{0^{+}} \times\langle g\rangle$ as $g$ centralizes $E$ (to see this, observe that $g$ is in the kernel $A_{(\delta)}$, and acts on every block $V_{i}$ as an element of $D_{2 n / u}$, whereas $E$ acts on $V_{i}$ as the center $Z\left(D_{2 n / u}\right)$.) We settle the lemma by proving the following equivalence :

$$
A_{0^{+}} \cong E_{0^{+}} \times \mathbb{Z}_{2} \Longleftrightarrow u \equiv 2 v(\bmod m / u)
$$

Suppose first that $A_{0^{+}}=E_{0^{+}} \times\langle g\rangle$, where $g \in A_{0^{+}}$and $g: 0^{-} \leftrightarrow u^{-}$. By (c) of Theorem 3.1, $\left\{v^{-},(v+m)^{-}\right\}^{A_{0^{+}}}=\left\{v^{-},(v+m)^{-}\right\}$. Applying Lemma 3.3 with $R=S$, $R_{*}=\{v, v+m\}$ and $r=v$, we obtain that the set $B=\left\{0^{+}, m^{+}, v^{-},(v+m)^{-}\right\}$is a block for $A$. The induced graph $\Gamma[B]$ is a 4 -circuit (again, see Figure 2). Denote by $A_{\{B\}}$ the setwise stabilizer of $B$ in $A$, and by $A_{\{B\}}^{B}$ the permutation group of $B$ induced by $A_{\{B\}}$. As $\Gamma[B]$ is a 4-circuit, $A_{\{B\}}^{B} \leq D_{8}$. This gives that $\left\{0^{+}, m^{+}\right\}$is a block for $A_{\{B\}}^{B}$, and therefore it is also a block for $A$. We conclude that $\delta_{m}=\left\{X: X \in \operatorname{Orb}\left(C^{m}, V\right)\right\}$ is a system of blocks for $A$. Consider the action of $A$ on $H\left(\mathbb{Z}_{m}, \eta_{n, m}(S)\right)$ defined in (3.1). Then $E \leq A_{\left(\delta_{m}\right)}$, while $g \notin A_{\left(\delta_{m}\right)}$. This implies that $g^{\delta_{m}}$ is an automorphism of $H\left(\mathbb{Z}_{m}, \eta_{n, m}(S)\right)$ which normalizes its canonical bicyclic group. This means that $g^{\delta_{m}}=$ $\varphi_{r, s, t}$ for some $r \in \mathbb{Z}_{m}^{*}$ and $s, t \in \mathbb{Z}_{m}$. Using that $g^{\delta_{m}}: 0^{+} \mapsto 0^{+}$and $0^{-} \mapsto \eta_{n, m}(u)^{-}$, we find that $s=0$ and $t=\eta_{n, m}(u)$, and so

$$
A^{\delta_{m}}=\left\langle D^{\delta_{m}}, \varphi_{r, 0, \eta_{n, m}(u)}\right\rangle .
$$

Also, $g^{\delta_{m}}: \eta_{n, m}(u)^{-} \mapsto 0^{-}$and $\eta_{n, m}(v)^{-} \mapsto \eta_{n, m}(v)^{-}$, hence $r \eta_{n, m}(u)=-\eta_{n, m}(u)$ and $r \eta_{n, m}(v)=\eta_{n, m}(v-u)$ hold in $\mathbb{Z}_{m}$. From these $r \equiv-1(\bmod m / u)$ and $r v \equiv$ $v-u(\bmod m / u)$, i.e., $u \equiv 2 v(\bmod m / u)$. The implication “ $\Rightarrow$ ” in (3.3) is now proved.

Suppose next that $u \equiv 2 v(\bmod m / u)$. Define the permutation $g$ of $V$ by

$$
g:(i v+j u)^{\varepsilon} \mapsto \begin{cases}(i v-(i+j) u)^{+} & \text {if } \varepsilon=+, \\ (i v-(i+j-1) u)^{-} & \text {if } \varepsilon=-,\end{cases}
$$

where $i \in\{0,1, \ldots, u-1\}$ and $j \in\{0,1, \ldots, n / u-1\}$. We complete the proof by verifying that $g \in A_{0^{+}}$. Since $0^{+g}=0^{+}$and $g: 0^{-} \leftrightarrow u^{-}$, this will imply that $A_{0^{+}}=$ $E_{0^{+}} \times\langle g\rangle$. Thus part " $\Leftarrow$ " of (3.3) is also proved.

Choose an arbitrary vertex $w \in \mathbb{Z}_{n}^{+}$such that $w=(i v+j u)^{+}, i \in\{0,1, \ldots, u-1\}$ and $j \in\{0,1, \ldots, n / u-1\}$, and suppose for the moment that $i<u-1$. Then $w$ has the following neighbors:

$$
(i v+j u)^{-},(i v+(j+1) u)^{-},((i+1) v+j u)^{-},((i+1) v+(j+m / u) u)^{-},
$$


where $v+1 \in\{0,1, \ldots, u-1\}$, and $j+1$ and $j+m / u$ are from $\{0,1, \ldots, n / u-1\}$. Thus these vertices are mapped by $g$ to

$(i v-(i+j-1) u)^{-},(i v-(i+j) u)^{-},((i+1) v-(i+j) u)^{-},((i+1) v-(i+j+m / u) u)^{-}$.

A direct check shows that these are just the neighbors of $w^{g}=(i v-(i+j) u)^{+}$. Let $i=u-1$. Then the neighbors of $w$ are:

$$
(i v+j u)^{-},(i v+(j+1) u)^{-},((j+v) u)^{-},((j+v+m / u) u)^{-},
$$

where $j+v$ and $j+v+m / u$ are from $\{0, \ldots, n / u-1\}$. Then these vertices are mapped by $g$ to

$$
(i v-(i+j-1) u)^{-},(i v-(i+j) u)^{-},(-(j+v-1) u)^{-},(-(j+v+m / u-1) u)^{-} .
$$

The first two are clearly connected with $w^{g}=(i v-(i+j) u)^{+}$; whereas the rest two are connected with $w^{g}$ if and only if the following equality holds in $\mathbb{Z}_{n}$ :

$\{i v-(i+j) u+v, i v-(i+j) u+v+m\}=\{-(j+v-1) u,-(j+v+m / u-1) u\}$.

Using that $v=u-1$, this reduces to $\{-(u-v) u,-(u-v) u+m\}=\{-v u,-v u+$ $m\}$. Finally, observe that this equality holds if $(u-v) u \equiv v u(\bmod m)$, and the latter congruence follows from the initial assumption that $u \equiv 2 v(\bmod m / u)$.

Lemma 3.5. Let $S$ be the set defined in Theorem 3.1. If $2 u \neq m$, then for the normalizer $N_{\text {Aut }\left(H\left(\mathbb{Z}_{n}, S\right)\right)}(C)$ of $C$ in $\operatorname{Aut}\left(H\left(\mathbb{Z}_{n}, S\right)\right)$,

$$
\left|\operatorname{Aut}\left(H\left(\mathbb{Z}_{n}, S\right)\right): N_{\operatorname{Aut}\left(H\left(\mathbb{Z}_{n}, S\right)\right)}(C)\right|= \begin{cases}2^{u-2} & \text { if } 2 \mid u \text { and }(u \neq 2 v(\bmod m / u) \text { or } \\ & u / 2 \equiv v(\bmod m / u)), \\ 2^{u-1} & \text { otherwise. }\end{cases}
$$

Proof. For short we set $A=\operatorname{Aut}\left(H\left(\mathbb{Z}_{n}, S\right)\right)$ and $N=N_{A}(C)$. Since $A=D A_{0^{+}}$and $D \leq N, N=D\left(N \cap A_{0}^{+}\right)$. The cases (1) and (2) in Lemma 3.4 are considered separately.

CASE 1. $u \not \equiv 2 v(\bmod m / u)$.

In this case, from Lemma 3.4, $A_{0^{+}}=E_{0^{+}}$, hence $|A|=2^{u} n$. Let $g \in N \cap A_{0}^{+}$. Since $g \in E_{0^{+}}$, it follows quickly that $g=1_{A}$ or $2 \mid u$ and $g=e_{1} e_{3} \cdots e_{u-1}$. Combining this with $N=D\left(N \cap G_{0}^{+}\right)$we find that $|N|=4 n$ if $2 \mid u$, and $|N|=2 n$ if $2 \nmid u$. Formula (3.5) follows.

CASE 2. $u \equiv 2 v(\bmod m / u)$.

From Lemma 3.4, $A_{0^{+}}=E_{0^{+}} \times F$ for a subgroup $F \leq A_{0^{+}},|F|=2$, hence $|A|=$ $2^{u+1} n$. It follows from the proof of Lemma 3.4 that, there exists $r \in \mathbb{Z}_{m}^{*}$ such that the following hold:

$$
r \eta_{n, m}(u)=-\eta_{n, m}(u) \text { and } r \eta_{n, m}(v)=\eta_{n, m}(v-u) .
$$


Let $s \in \mathbb{Z}_{n}^{*}$ such that $\eta_{n, m}(s)=r$. Then

$$
s u \in\{-u,-u+m\} \text { and } s v \in\{v-u, v-u+m\} .
$$

Suppose that $2 \nmid u$. Then we get as before that $N \cap E_{0^{+}}$is trivial. Notice also that, $u \equiv 2 v+m / u(\bmod n / u)$, which follows from the assumption that $u \equiv 2 v(\bmod m / u)$ and that $2 \nmid u$. Thus $2 \nmid m$ and $2 \mid(u+m)$, implying that in (3.6) we have $s u=-u$. We obtain that $\varphi_{s, u, 0} \in N \cap\left(A_{0^{+}} \backslash E_{0^{+}}\right)$, and so $\left|N \cap A_{0^{+}}\right|=2$.

Suppose next that $2 \mid u$. Then $\left|N \cap E_{0^{+}}\right|=2$. It is easily seen that $\left|N \cap A_{0^{+}}\right|=4$ if and only if there exists $r \in \mathbb{Z}_{n}^{*}$ such that $r u=-u$ and $r v=v-u$ hold in $\mathbb{Z}_{n}$. Consider the following system of linear congruences:

$$
x u \equiv u(\bmod n), x v \equiv v-u(\bmod n) .
$$

From the first congruence we can write $x$ in the form $x=y n / u-1$. Substitute this into the second congruence. We obtain that $y v n / u \equiv 2 v-u(\bmod n)$. This has a solution if and only if $\operatorname{gcd}(v n / u, n) \mid(2 v-u)$. Suppose that $\operatorname{gcd}(v, n) \neq 1$. Using that $\langle u, v\rangle=\mathbb{Z}_{n}$ and that $2 \mid u$, we obtain that $\operatorname{gcd}(v, m / u) \neq 1$. However, then from the assumption that $u \equiv 2 v(\bmod m / u)$ it follows that also $\operatorname{gcd}(v, u) \neq 1$, which contradicts that $\langle u, v\rangle=\mathbb{Z}_{n}$. Hence $\operatorname{gcd}(v, n)=1, \operatorname{gcd}(v n / u, n)=n / u$, and so (3.7) has a solution if and only if $u \equiv$ $2 v(\bmod n / u)$, or equivalently, $u / 2 \equiv v(\bmod m / u)($ recall that $2 \mid u$ and $u \mid m)$. It is not hard to show that any solution to (3.7) is necessarily prime to $n$, hence is in $\mathbb{Z}_{n}^{*}$. The above arguments can be summarized as follows: $|N|=8 n$ if $2 \mid u$ and $u / 2 \equiv v(\bmod m / u)$, and $|N|=4 n$ otherwise. This is consistent with (3.5). The lemma is proved.

Lemma 3.6. Let $r \in \mathbb{Z}_{n}^{*}, r \neq 1$ and $s \in \mathbb{Z}_{n}$ such that the permutation $\varphi_{r, 0, s}$ is of order 2. Then the group $\left\langle D, \varphi_{r, 0, s}\right\rangle$ contains a bicyclic subgroup different from $C$ if and only if $8 \mid n, r=n / 2+1$, and $s=0$ or $s=n / 2$.

Proof. Suppose that $\left\langle D, \varphi_{r, 0, s}\right\rangle$ contains a bicyclic subgroup $X$ such that $X \neq C$. Then $X$ is generated by a permutation in the form $c^{i} \varphi_{r, 0, s}$. Since $\varphi_{r, 0, s}^{2}=i d_{V}, r^{2}=1$ in $\mathbb{Z}_{n}$, and we calculate that $\left(c^{i} \varphi_{r, 0, s}\right)^{2}$ sends $x^{+}$to $(x+r(r+1) i)^{+}$for every $x \in \mathbb{Z}_{n}$. That $\mathbb{Z}_{n}^{+}$is an orbit of $X$ is equivalent to the condition that $\operatorname{gcd}(n, r+1)=2$. Using this and that $r^{2}-1=(r-1)(r+1) \equiv 0(\bmod n)$, we find that $n / 2$ divides $r-1$, so $r=1$ or $r=n / 2+1$. Since $r \neq 1$, we have that $r=n / 2+1$ and $8 \mid n$. Then $\left(\varphi_{r, 0, s}\right)^{2}$ sends $x^{-}$ to $(x+(n / 2+2) s)^{-}$. Since $\left(\varphi_{r, 0, s}\right)^{2}=i d_{V}$, we obtain that $s=0$ or $s=n / 2$.

On the other hand, it can be directly checked that, if $8 \mid n, r=n / 2+1$ and $s \in$ $\{0, n / 2\}$, then the permutation $c \varphi_{r, 0, s}$ generates a bicyclic subgroup of $\left\langle D, \varphi_{r, 0, s}\right\rangle$. Obviously, this bicyclic subgroup cannot be $C$. The lemma is proved.

Everything is prepared to prove the main result of the section.

Proof of Theorem 3.1. The case that $2 u=m$ is settled already in Lemma 3.2, hence let $2 u \neq m$. We consider the action of $A=\operatorname{Aut}\left(H\left(\mathbb{Z}_{n}, S\right)\right)$ on the system of blocks $\delta_{m}$ defined in (3.1). We claim that the corresponding image $A^{\delta_{m}}$ has a unique bicyclic subgroup (which is, of course, $C^{\delta_{m}}$ ).

This is easy to see if $A_{0^{+}}=E_{0^{+}}$, because in this case $A^{\delta_{m}}=\left(D A_{0^{+}}\right)^{\delta_{m}}=D^{\delta_{m}}$.

Let $A_{0^{+}} \neq E_{0^{+}}$. Then $A_{0^{+}}=E_{0^{+}} \times F$ for some subgroup $F,|F|=2$. By (3.4), $A^{\delta_{m}}=\left\langle D^{\delta_{m}}, \varphi_{r, 0, \eta_{n, m}(u)}\right\rangle$. Also, $r \equiv-1(\bmod m / u)$, hence $r \neq 1$ in $\mathbb{Z}_{m}$. By Lemma $3.5, A^{\delta_{m}}$ contains more than one bicyclic subgroup if and only if $8 \mid m, r=m / 2+1$ and 
$\eta_{n, m}(u) \in\{0, m / 2\}$. In the latter case $u \in\{m, m / 2\}$, which is impossible as $u<m / 2$. Hence $A^{\delta_{m}}$ contains indeed a unique bicyclic subgroup.

We calculate next the number of bicyclic groups of $H\left(\mathbb{Z}_{n}, S\right)$; we denote this number by $\mathbb{B}$. In fact, we are going to derive the following formula:

$$
\mathbb{B}= \begin{cases}2^{u-2} & \text { if } 2 \mid u \text { and } 2 \nmid(m / u), \\ 2^{u-1} & \text { otherwise. }\end{cases}
$$

Let $g \in G$ such that $\langle g\rangle$ is a bicyclic group of $H\left(\mathbb{Z}_{n}, S\right)$. Since $G=D A_{0^{+}}, g$ can be written as $g=x y$ with $x \in D$ and $y \in A_{0^{+}}$. Since $\langle g\rangle$ is a bicyclic group, $g$ fixes the color classes setwise, implying that $x \in C$. The image $\langle g\rangle^{\delta_{m}}$ is also a bicyclic subgroup of $A^{\delta_{m}}$, hence by the previous paragraph, $\langle g\rangle^{\delta_{m}}=C^{\delta_{m}}$. Now, since $x \in C, y^{\delta_{m}} \in C^{\delta_{m}}$, from which $y^{\delta_{m}}=i d_{\delta_{m}}$. We conclude that $x=c^{i} \in C$ for some $i \in\{1, \ldots, n-1\}$ with $\operatorname{gcd}(i, m)=1$, and $y \in E_{0^{+}}$, and so $y=e_{I}$ for a subset $I \subseteq\{1, \ldots, u-1\}$. Obviously, the product $\phi(n) \mathbb{B}$ calculates the number of elements $g \in G$ such that $\langle g\rangle$ is a bicyclic group of $H\left(\mathbb{Z}_{n}, S\right)$, where $\phi$ denotes the Euler's totient function. Therefore, $\phi(n) \mathbb{B}$ is equal to the number of elements in the form $c^{i} e_{I}$ that $i \in\{1, \ldots, n-1\}, \operatorname{gcd}(i, m)=1$, $I \subseteq\{1, \ldots, u-1\}$, and $\left\langle c^{i} e_{I}\right\rangle$ is a bicyclic group of $H\left(\mathbb{Z}_{n}, S\right)$.

Let us pick $c^{i} e_{I}$ with $i \in\{1, \ldots, n-1\}, \operatorname{gcd}(i, m)=1$, and $I \subseteq\{1, \ldots, u-1\}$. It is easily seen that $e_{I} c^{i}=c^{i} e_{I+i}$, where $I+i=\{x+i: x \in I\}$, here the addition is taken modulo $u$. Using this and induction on $u$, it follows that

$$
\left(c^{i} e_{I}\right)^{u}=c^{u i} e_{I} e_{I+i} \cdots e_{I+(u-1) i} .
$$

Since $\operatorname{gcd}(i, m)=1$ and $u \mid m, \operatorname{gcd}(i, u)=1$, from which

$$
e_{I} e_{I+i} \cdots e_{I+(u-1) i}=\left(e_{0} e_{1} \cdots e_{u-1}\right)^{|I|}=c^{m|I|} .
$$

Thus $\left(c^{i} e_{I}\right)^{u}=c^{u\left(i+\frac{m}{u}|I|\right)}$. This and $\operatorname{gcd}(i, u)=1$ show that $\left\langle c^{i} e_{I}\right\rangle$ is a semiregular group. Therefore, $\left\langle c^{i} e_{I}\right\rangle$ is a bicyclic group if and only if $c^{i} e_{I}$ is of order $n$, or equivalently,

$$
\operatorname{gcd}\left(i+\frac{m}{u}|I|, \frac{2 m}{u}\right)=1 .
$$

Notice that, $\operatorname{since} \operatorname{gcd}(i, m)=1$, the greatest common divisor above is always equal to 1 or 2. Suppose at first that $2 \mid(m / u)$. Then $2 \mid m$ and $i$ is odd. Hence (3.9) always holds. We obtain that the number of elements in $A$ which generate a bicyclic group is $\phi(n) 2^{u-1}$, and so $\mathbb{B}=2^{u-1}$, as claimed in (3.8). Suppose next that $2 \nmid(m / u)$. Now, if $2 \mid u$, then $2 \mid m$, hence $2 \nmid i$, and so (3.9) holds if and only if $|I|$ is even. We deduce from this that $\mathbb{B}=2^{u-2}$, as claimed in (3.8). Finally, if $2 \nmid u$, then $2 \nmid m$, and in this case (3.9) holds if and only if $\operatorname{gcd}(i, n)=1$ and $|I|$ is even, or $\operatorname{gcd}(i, n)=2$ and $|I|$ is odd. We calculate that $\mathbb{B}=2^{u-1}$, and this completes the proof of (3.8).

Let $\Xi$ be a bicyclic base of $H\left(\mathbb{Z}_{n}, S\right)$. By (3.5) and (3.8) we obtain that, $|\Xi|>1$ if and only if

$$
\left|A: N_{A}(C)\right|=2^{u-2} \text { and } \mathbb{B}=2^{u-1} .
$$

This happens exactly when

$(2 \mid u$ and $(u \not \equiv 2 v(\bmod m / u)$ or $u / 2 \equiv v(\bmod m / u)))$ and $(2 \nmid u$ or $2 u \mid m)$. 
After some simplification,

$$
|\Xi|>1 \Longleftrightarrow 2|u, 2 u| m \text { and } u / 2 \not \equiv v+m /(2 u)(\bmod m / u) .
$$

Suppose that $|\Xi|>1$. Then $A$ contains exactly $2^{n-1}$ bicyclic subgroups, $2^{n-2}$ of which are conjugate to $C$. These $2^{n-1}$ subgroups are enumerated as: $\left\langle c e_{I}\right\rangle, I \subseteq\{1, \ldots, u-1\}$. For $i \in\{1, \ldots, u-2\}, e_{i} c e_{i}=c e_{\{i, i+1\}}$. We can conclude that the bicyclic subgroups split into two conjugacy classes:

$$
\left\{\left\langle c e_{I}\right\rangle: I \subseteq\{1, \ldots, u-1\},|I| \text { is even }\right\} \text { and }\left\{\left\langle c e_{I}\right\rangle: I \subseteq\{1, \ldots, u-1\},|I| \text { is odd }\right\} .
$$

In particular, $|\Xi|=2$. Choose $\xi$ from $\operatorname{Sym}(V)$ which satisfies

$$
\xi c \xi^{-1}=c e_{1} \text { and } \xi: 0^{+} \mapsto 0^{+}, 0^{-} \mapsto 0^{-} .
$$

Then $\Xi$ can be chosen as $\Xi=\left\{i d_{V}, \xi\right\}$. Also, $\left\{v^{-},(v+m)^{-}\right\}^{\xi}=\left\{v^{-},(v+m)^{-}\right\}$, and since $\left(c e_{1}\right)^{u+m}=c^{u},\left(u^{-}\right)^{\xi}=\left(0^{-}\right)^{\left(c e_{1}\right)^{u+m} \xi}=\left(0^{-}\right)^{\xi c^{u+m}}=(u+m)^{-}$. Thus $H\left(\mathbb{Z}_{n}, S\right)^{\xi}=H\left(\mathbb{Z}_{n},\{0, u+m, v, v+m\}\right)$. The theorem follows from Theorem 2.6.

\section{Proof of Theorem 1.1}

Theorem 1.1 follows from Theorem 3.1 and the following theorem.

Theorem 4.1. Let $H\left(\mathbb{Z}_{n}, S\right)$ be a connected Haar graph such that $|S|=4$ and $S$ is not a BCI-subset. Then $n=2 m$, and there exist $a \in \mathbb{Z}_{n}^{*}$ and $b \in \mathbb{Z}_{n}$ such that $a S+b=$ $\{0, u, v, v+m\}$ and the conditions $(a)-(c)$ in Theorem 3.1 hold.

Before we prove Theorem 4.1 it is necessary to give three preparatory lemmas. For an element $i \in \mathbb{Z}_{n}$, we denote by $|i|$ the order of $i$ viewed as an element of the additive group $\mathbb{Z}_{n}$. Thus we have $|i|=n / \operatorname{gcd}(n, i)$.

Lemma 4.2. If $R=\{i, n-i, j\}$ is a generating subset of $\mathbb{Z}_{n}$ with $|i|$ odd, then $R$ is a CI-subset.

Proof. For short we set $A=\operatorname{Aut}\left(\operatorname{Cay}\left(\mathbb{Z}_{n}, R\right)\right)$ and denote by $A_{0}$ the stabilizer of $0 \in \mathbb{Z}_{n}$ in $A$. Clearly, $A_{0}$ leaves $R$ setwise fixed. If $A_{0}$ acts on $R$ trivially, then $A \cong \mathbb{Z}_{n}$, and the lemma follows by Theorem 2.1. If $A_{0}$ acts on $R$ transitively, then $\operatorname{Cay}\left(\mathbb{Z}_{n}, R\right)$ is edgetransitive. This condition forces that $R$ is a CI-subset (see [10, page 320]).

We are left with the case that $R$ consists of two orbits under $A_{0}$. These orbits must be $\{i, n-i\}$ and $\{j\}$. It is clear that $A_{0}$ leaves the subgroups $\langle i\rangle$ and $\langle j\rangle$ fixed; moreover, the latter set is fixed pointwise, and since $|i|$ is odd, $\langle i\rangle$ consists of $(|i|-1) / 2$ orbits under $A_{0}$, each of length 2 , and one orbit of length 1 . We conclude that $\mathbb{Z}_{n}=\langle i\rangle \times\langle j\rangle$, and also that $A$ is permutation isomorphic to the permutation direct product $\left(\left(\mathbb{Z}_{|i|}\right)_{\text {right }} \rtimes\langle\pi\rangle\right) \times\left(\mathbb{Z}_{|j|}\right)_{\text {right }}$. For $\ell \in\{|i|,|j|\},\left(\mathbb{Z}_{\ell}\right)_{\text {right }}$ is generated by the affine transformation $x \mapsto x+1$, and $\pi$ is the affine transformation $x \mapsto-x$. We leave for the reader to verify that the above group has a unique regular cyclic subgroup. The lemma follows by Theorem 2.1.

Lemma 4.3. Let $n=2 m$ and $R=\{i, n-i, j, j+m\}$ be a subset of $\mathbb{Z}_{n}$ such that

(a) $R$ generates $\mathbb{Z}_{n}$;

(b) $|i|$ is odd; 
(c) the stabilizer $\operatorname{Aut}\left(\operatorname{Cay}\left(\mathbb{Z}_{n}, R\right)\right)_{0}$ leaves the set $\{i, n-i\}$ setwise fixed.

Then $R$ is a CI-subset.

Proof. For short we set $A=\operatorname{Aut}\left(\operatorname{Cay}\left(\mathbb{Z}_{n}, R\right)\right)$. Let $T$ be a subset of $\mathbb{Z}_{n}$ such that $\operatorname{Cay}\left(\mathbb{Z}_{n}, R\right) \cong \operatorname{Cay}\left(\mathbb{Z}_{n}, T\right)$ and let $f$ be an isomorphism from $\operatorname{Cay}\left(\mathbb{Z}_{n}, R\right)$ to $\operatorname{Cay}\left(\mathbb{Z}_{n}, T\right)$ such that $f(0)=0$. Let us consider the subgraphs

$$
\Gamma_{1}=\operatorname{Cay}\left(\mathbb{Z}_{n},\{i, n-i\}\right) \text { and } \Gamma_{2}=\operatorname{Cay}\left(\mathbb{Z}_{n},\{j, j+m\}\right) .
$$

By condition $(c)$, the group $A$ preserves both of these subgraphs, that is, $A \leq \operatorname{Aut}\left(\Gamma_{\ell}\right)$ for $\ell \in\{1,2\}$. As $f$ is an isomorphism between two Cayley graphs, $f\left(\mathbb{Z}_{n}\right)_{\text {right }} f^{-1} \leq$ $A$. Then $f\left(\mathbb{Z}_{n}\right)_{\text {right }} f^{-1} \leq A \leq \operatorname{Aut}\left(\Gamma_{\ell}\right)$, implying that $f$ maps $\Gamma_{\ell}$ to a Cayley graph $\operatorname{Cay}\left(\mathbb{Z}_{n}, T_{\ell}\right)$ for both $\ell \in\{1,2\}$. Clearly, $T=T_{1} \cup T_{2}$. It was proved by Sun [15] (see also [10]) that every subset of $\mathbb{Z}_{n}$ of size 2 is a CI-set. Using this, it follows from $\operatorname{Cay}\left(\mathbb{Z}_{n},\{i, n-i\}\right) \cong \operatorname{Cay}\left(\mathbb{Z}_{n}, T_{1}\right)$ that $T_{1}=a\{i, n-i\}$ for some $a \in \mathbb{Z}_{n}^{*}$. Letting $t_{1}=a i$, we have $T_{1}=\left\{t_{1}, n-t_{1}\right\}$ such that $|i|=\left|t_{1}\right|$. In the same way, $T_{2}=a^{\prime}\{j, j+m\}$ for some $a^{\prime} \in \mathbb{Z}_{n}^{*}$, and letting $t_{2}=a^{\prime} j$, we have $T_{2}=\left\{t_{2}, t_{2}+m\right\}$ with $\left|t_{2}\right|=|j|$. Since $f(0)=0, f$ maps $\{i, n-i\}$ to $T_{1}=\left\{t_{1}, n-t_{1}\right\}$ and $\{j, j+m\}$ to $T_{2}=\left\{t_{2}, t_{2}+m\right\}$.

We claim that the partition of $\mathbb{Z}_{n}$ into the cosets of $\langle m\rangle$ is a system of blocks for $\operatorname{Aut}\left(\Gamma_{2}\right)$, hence also for the group $A \leq \operatorname{Aut}\left(\Gamma_{2}\right)$. Let us put $\bar{A}=\operatorname{Aut}\left(\Gamma_{2}\right)$. Then $\bar{A}_{0}$ leaves the set $T=\{j, j+m\}$ setwise fixed. Thus the setwise stabilizer $\bar{A}_{\{T\}}$ of the set $T$ in $\bar{A}$ can be written as $\bar{A}_{\{T\}}=\bar{A}_{\{T\}} \cap \bar{A}=\bar{A}_{\{T\}} \cap \bar{A}_{0}\left(\mathbb{Z}_{n}\right)_{\text {right }}=\bar{A}_{0}\left(\bar{A}_{\{T\}} \cap\left(\mathbb{Z}_{n}\right)_{\text {right }}\right)=$ $\bar{A}_{0}\left\langle m_{\text {right }}\right\rangle$. Here $\left(\mathbb{Z}_{n}\right)_{\text {right }}$ is generated by the affine transformation $x \mapsto x+1$, and $m_{\text {right }}$ is the permutation $x \mapsto x+m$ for every $x \in \mathbb{Z}_{n}$. Thus $\bar{A}_{0}\left\langle m_{\text {right }}\right\rangle$ is a subgroup of $\bar{A}$ which clearly contains $\bar{A}_{0}$. By [5, Theorem $\left.1.5 \mathrm{~A}\right]$, the orbit of 0 under the group $\bar{A}_{0}\left\langle m_{\text {right }}\right\rangle$ is a block for $\bar{A}$. Now, the required statement follows as the latter orbit is equal to $0^{\bar{A}_{0}\left\langle m_{\mathrm{r} i g h t}\right\rangle}=0^{\left\langle m_{\mathrm{r} i g h t}\right\rangle}=\langle m\rangle$.

Since the partition of $\mathbb{Z}_{n}$ into the cosets of $\langle m\rangle$ is a system of blocks for $A$, the isomorphism $f$ induces an isomorphism from $\operatorname{Cay}\left(\mathbb{Z}_{m}, \eta_{n, m}(R)\right)$ to $\operatorname{Cay}\left(\mathbb{Z}_{m}, \eta_{n, m}(T)\right)$, we denote this isomorphism by $\bar{f}$. Note that, $\bar{f}(0)=0$ for the identity element $0 \in \mathbb{Z}_{m}$.

The set $\eta_{n, m}(R)$ satisfies the conditions (a)-(c) of Lemma 4.2, hence it is a CI-subset. This means that $\bar{f}$ is equal to a permutation $x \mapsto r x$ for some $r \in \mathbb{Z}_{m}^{*}$. Let $s \in \mathbb{Z}_{n}^{*}$ such that $\eta_{n, m}(s)=r$. Then $\eta_{n, m}(s i)=\eta_{n, m}(s) \eta_{n, m}(i)=\eta_{n, m}\left(t_{1}\right)$, and so the following holds in $\mathbb{Z}_{n}$ :

$$
s i=t_{1} \text { or } s i=t_{1}+m \text {. }
$$

The order $\left|t_{1}\right|=|i|$ is odd by (b), implying that $\left|t_{1}\right| \neq\left|t_{1}+m\right|$, and so $s i=t_{1}$ holds in (4.1). We conclude that $s R=T$, so $R$ is a CI-subset. The lemma is proved.

Lemma 4.4. Let $n=2 m$ and $S=\{0, u, v, v+m\}$ such that

(a) S generates $\mathbb{Z}_{n}$;

(b) $1<u<n, u \mid n$ but $u \nmid m$;

(c) $\operatorname{Aut}\left(H\left(\mathbb{Z}_{n}, S\right)\right)_{0^{+}}$leaves the set $\left\{0^{-}, u^{-}\right\}$setwise fixed.

Then $S$ is a BCI-subset. 
Proof. Let $\delta$ be the partition of $V$ defined in (3.2). Applying Lemma 3.3 with $R=S$, $R_{*}=\{0, u\}$ and $r=0$, we obtain that $\delta$ is a system of blocks for $A=\operatorname{Aut}\left(H\left(\mathbb{Z}_{n}, S\right)\right)$. Thus the stabilizer $A_{0+}$ leaves the set $V_{0}$ setwise fixed, and we may consider the action of $A_{0^{+}}$on $V_{0}$. The subgraph of $H\left(\mathbb{Z}_{n}, S\right)$ induced by the set $V_{0}$ is a circuit of length $2 n / u$, thus $A_{0^{+}}$fixes also the vertex on this circuit antipodal to $0^{+}$. We find that this antipodal vertex is $(u / 2+m)^{-}$. Therefore, $A_{0^{+}}=A_{(m+u / 2)^{-}}$, and thus $S$ is a BCI-subset if and only if $S-u / 2+m$ is a CI-subset of $\mathbb{Z}_{n}$, see Proposition 2.8. The latter set is

$$
S-u / 2+m=\{u / 2+m,-u / 2+m, v-u / 2, v-u / 2+m\} .
$$

Since $u \nmid m, u$ is even and the order $|u / 2+m|$ is odd. Lemma 4.3 is applicable to the set $S-u / 2+m$ (choose $i=u / 2+m$ and $j=v-u / 2$ ), it gives us that $S-u / 2+m$ is a CI-subset. This completes the proof.

Proof of Theorem 4.1. Let $S$ be the subset of $\mathbb{Z}_{n}$ given in Theorem 4.1. We deal first with the case when the canonical bicyclic group $C$ is normal in $A=\operatorname{Aut}\left(H\left(\mathbb{Z}_{n}, S\right)\right)$.

CASE $1 . C \unlhd A$.

By Theorem 2.6, there is a bicyclic group $X$ of $H\left(\mathbb{Z}_{n}, S\right)$ such that $X \neq C$. Since $C \unlhd$ $A, X$ is generated by a permutation in the form $c^{i} \varphi_{r, 0, s}, r \in \mathbb{Z}_{n}^{*}, s \in \mathbb{Z}_{n}$, and ord $\left(\varphi_{r, 0, s}\right) \geq$ 2. The permutation $\varphi_{r, 0, s}$ acts on both $\mathbb{Z}_{n}^{+}$and $\mathbb{Z}_{n}^{-}$as an affine transformation. This fact together with the connectedness of $H\left(\mathbb{Z}_{n}, S\right)$ imply that, $\varphi_{r, 0, s}$ acts faithfully on $S^{-}$. Thus $\operatorname{ord}\left(\varphi_{r, 0, s}\right) \leq 4$.

Suppose that $\operatorname{ord}\left(\varphi_{r, 0, s}\right)=4$. We may assume without loss of generality that $S^{-}$can be obtained as $S^{-}=\left\{\left(0^{-}\right)^{\varphi_{r, 0, s}^{j}}: j \in\{0,1,2,3\}\right\}$, and so $S=\left\{0, s,(r+1) s,\left(r^{2}+\right.\right.$ $r+1) s\}$ and $\left(r^{3}+r^{2}+r+1\right) s=0$. Since $H\left(\mathbb{Z}_{n}, S\right)$ is connected, $\operatorname{gcd}(s, n)=1$, and $(r+1)\left(r^{2}+1\right)=0$. We find that $\left(c^{i} \varphi_{r, 0, s}\right)^{4}$ sends $x^{+}$to $\left(x+r(r+1)\left(r^{2}+1\right) i\right)^{+}=x^{+}$. Since $X=\left\langle c^{i} \varphi_{r, 0, s}\right\rangle$ is bicyclic, $n=4$, and so $H\left(\mathbb{Z}_{n}, S\right) \cong K_{4,4}$. This, however, contradicts that $C \unlhd A$.

Now, suppose that $\operatorname{ord}\left(\varphi_{r, 0, s}\right)=3$. If $A_{0^{+}}$is transitive on $S^{-}$, then it must be regular [9, Theorem 4.3]. This implies that $S^{-}$splits into two orbits under $A_{0^{+}}$with length 1 and 3 , respectively. Let $s \in S$ such that $\left\{s^{-}\right\}$is an orbit under $A_{0^{+}}$. Then $A_{0^{+}}=A_{s^{-}}$, and by Proposition 2.8, $S-s$ is not a CI-subset of $\mathbb{Z}_{n}$. However, in this case the graph $\operatorname{Cay}\left(\mathbb{Z}_{n}, S-s\right)$ is edge-transitive, and thus $S-a$ is a CI-subset (see [10, page 320]), which is a contradiction.

Finally, suppose that $\operatorname{ord}\left(\varphi_{r, 0, s}\right)=2$. If $r=1$, then $2 \mid n$ and $s=m$, where $n=2 m$. This implies that $S^{-}$is a union of two orbits of $C^{m}$, we may write $S=\{0, m, s, s+m\}$. The graph $H\left(\mathbb{Z}_{n}, S\right)$ is then isomorphic to the lexicographical product $C_{n}\left[K_{2}^{c}\right]$ of an $n$ circuit $C_{n}$ with the graph $K_{2}^{c}$, see Figure 3 . It is easily seen that then $A_{0^{+}}$is not faithful on the set $S^{-}$, which is a contradiction.

Let $r \neq 1$. By Lemma 3.6, $8 \mid n, r=m+1$ and $s \in\{0, m\}$, where $n=2 m$. We consider only the case when $s=0$ (the case when $s=m$ can be treated in the same manner). Then $\mathbb{Z}_{n}^{-}$splits into the following orbits under $\varphi_{r, 0, s}$ :

$$
\left\{(2 i)^{-}\right\},\left\{(2 i+1)^{-},(2 i+1+m)^{-}\right\}, \text {where } i \in\{0,1, \ldots, m-1\} .
$$

Since $H\left(\mathbb{Z}_{n}, S\right)$ is connected and cannot be the union $C^{m}$-orbits (see above), $S^{-}$contains one orbit under $\varphi_{r, 0, s}$ of length 2 , and two orbits of length 1 . Let $S_{1}$ denote the orbit of 


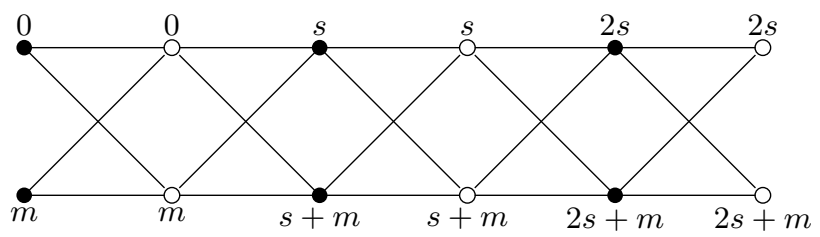

Figure 3: The lexicographical product $C_{n}\left[K_{2}^{c}\right]$.

length 2 and let $S_{2}=S \backslash S_{1}$. Then we may write $S_{1}=\{s, s+m\}$, and $S_{2}=\left\{s^{\prime}, s^{\prime \prime}\right\}$, where both $s^{\prime}$ and $s^{\prime \prime}$ are even. Let $u=\operatorname{gcd}\left(s^{\prime}-s^{\prime \prime}, n\right)$. Then $u$ is a divisor of $n$ and also $2 \mid u$. There exist $a \in \mathbb{Z}_{n}^{*}$ such that $a\left(s^{\prime}-s^{\prime \prime}\right) \equiv u(\bmod n)$. Choosing $b=-a s^{\prime \prime}$ (all arithmetic is done in $\mathbb{Z}_{n}$ ), we find that $a S_{2}+b=\{u, 0\}$. Now, letting $v=a s+b$, we get $a S_{1}+b=\{v, v+m\}$. We finish the proof of this case by showing that the set $R=a S+b=\{0, u, v, v+m\}$ satisfies the conditions (a)-(c) of Theorem 3.1.

(a): As $H\left(\mathbb{Z}_{n}, S\right)$ is connected, $H\left(\mathbb{Z}_{n}, R\right)$ is also connected. This implies that $\{u, v\}$ is a generating set of $\mathbb{Z}_{n}$.

(c): Since $C \unlhd A, C \unlhd \operatorname{Aut}\left(H\left(\mathbb{Z}_{n}, R\right)\right)$. To the contrary assume that the stabilizer $\operatorname{Aut}\left(H\left(\mathbb{Z}_{n}, R\right)\right)_{0^{+}}$does not leave $\left\{0^{-}, u^{-}\right\}$setwise fixed. Thus there exists some $g \in A_{0^{+}}$ which maps $v^{-}$into $\left\{0^{-}, u^{-}\right\}$. Letting $w_{1}^{-}=\left(v^{-}\right)^{g}$ and $w_{2}^{-}=\left((v+m)^{-}\right)^{g}$, we find that $w_{1}-w_{2}=m$, and from this that $u=m$. However, then $H\left(\mathbb{Z}_{n}, R\right) \cong C_{n}\left[K_{2}^{c}\right]$, which we have already excluded above. Thus $\operatorname{Aut}\left(H\left(\mathbb{Z}_{n}, R\right)\right)_{0^{+}}$fixes setwise $\left\{0^{-}, u^{-}\right\}$.

(b): We have already showed (see previous paragraph) that $u \neq m$ and $1<u$. Since $S$ is not a BCI-subset, $R$ is also a not a BCI-subset. This also implies that $u \mid m$ by Lemma 4.4, and we conclude that $1<u<m$ and $u \mid m$, as required.

CASE 2. $C \unlhd A$.

Let $A_{0^{+}}$act transitively on $S^{-}$. This gives that $H\left(\mathbb{Z}_{n}, S\right)$ is edge-transitive. Since $C \unlhd$ $A, D \unlhd A$, in other words, $H\left(\mathbb{Z}_{n}, S\right)$ is non-normal as a Cayley graph over the dihedral group $D$. We apply [8, Theorem 1.2], and obtain that $H\left(\mathbb{Z}_{n}, S\right)$ is either isomorphic to $K_{n}\left[K_{2}^{c}\right]$, or to one of 5 graphs of orders $10,14,26,28$ and 30 , respectively. Suppose that the former case holds. Then $n=2 m$, and we obtain quickly that $S$ consists of two $C^{m}$ orbits. Then $S$ can be mapped by an affine transformation to a set $\{0, m, v, v+m\}$, where $\langle m, v\rangle \cong \mathbb{Z}_{n}$. Then $v$ or $v+m$ is a generating element of $\mathbb{Z}_{n}$, and so $S$ can actually be mapped by an affine transformation to $\{0, m, 1,1+m\}$. Now, the same holds for any set $T$ with $H\left(\mathbb{Z}_{n}, T\right) \cong H\left(\mathbb{Z}_{n}, S\right) \cong K_{n}\left[K_{2}^{c}\right]$, contradicting that $S$ is not a BCI-subset. In the latter case, a direct computation by the computer package MAGMA [4] shows that none of these graphs is possible (in fact, in each case the corresponding subset $S$ is a BCI-subset).

The set $S^{-}$cannot split into two orbits under $A_{0^{+}}$of size 1 and 3 , respectively (see the argument above). We are left with the case that $S=S_{1} \cup S_{2},\left|S_{1}\right|=\left|S_{2}\right|$, and $A_{0^{+}}$leaves both sets $S_{1}$ and $S_{2}$ setwise fixed. For $i \in\{1,2\}$, let $n_{i}=\left|\left\langle S_{i}-S_{i}\right\rangle\right|, n_{1} \leq n_{2}$, where $S_{i}-S_{i}=\left\{a-b: a, b \in S_{i}\right\}$.

We claim that $n_{1}=2$. To the contrary assume that $n_{1}>2$. We prove first that $C^{n / n_{1}} \unlhd A$. Applying Lemma 3.3 with $R=S, R_{*}=S_{1}$ and $r=s_{1} \in S_{1}$, we obtain that the partition

$$
\delta=\left\{X \cup X^{\psi_{1, s_{1},-s_{1}}}: X \in \operatorname{Orb}\left(C^{n / n_{1}}, V\right)\right\},
$$


is a system of blocks for $A$. Let us consider the action of $A_{(\delta)}$ (the kernel of $A$ acting on $\delta$ ) on the block of $\delta$ which contains $0^{+}$. Denote this block by $\Delta$, and by $\Delta^{\prime}$ the block which contains $s^{-}$for some $s \in S_{2}$. Notice that, the subgraph of $H\left(\mathbb{Z}_{n}, S\right)$ induced by any block of $\delta$ is a circuit of length $2 n_{1}$, and when deleting these circuits, the rest splits into pairwise disjoint circuits of length $2 n_{2}$. Let $\Sigma$ denote the unique $\left(2 n_{2}\right)$-circuit through $s^{-}$. Now, suppose that $g \in A_{(\delta)}$ which fixes $\Delta$ pointwise. If $V(\Sigma) \cap \Delta=\left\{0^{+}\right\}$, then $g$ must fix the edge $\left\{0^{+}, s^{-}\right\}$, and so fixes also $s^{-}$. If $V(\Sigma) \cap \Delta \neq\left\{0^{+}\right\}$, then $|V(\Sigma) \cap \Delta|=n_{2}>2$. This implies that $g$ fixes every vertex on $\Sigma$, in particular, also $s^{-}$. The block $\Delta^{\prime}$ has at least $n_{1}$ vertices having a neighbor in $\Delta$, hence by the previous argument we find that all are fixed by $g$. Since $n_{1}>2, \Delta^{\prime}$ is fixed pointwise by $g$. It follows, using the connectedness of $H\left(\mathbb{Z}_{n}, S\right)$, that $g=i d_{V}$, hence that $A_{(\delta)}$ is faithful on $\Delta$. Thus $C^{n / n_{1}}$ is a characteristic subgroup of $A_{(\delta)}$, and since $A_{(\delta)} \unlhd A, C^{n / n_{1}} \unlhd A$.

Let $G$ be the unique normal subgroup of $A$ that fixes the color classes $\mathbb{Z}_{n}^{+}$and $\mathbb{Z}_{n}^{-}$. We consider $N=G \cap C_{A}\left(C^{n / n_{1}}\right)$. Then $C \leq N$ and $N \unlhd A$. Pick $g \in N_{0^{+}}$such that $g$ acts non-trivially on $S^{-}$. Since $N$ centralizes $C^{n / n_{1}}, g$ fixes pointwise the orbit of $0^{+}$under $C^{n / n_{1}}$, and hence also $\Delta$. Then $g^{2}$ fixes $S^{-}$pointwise, and so also $\Delta^{\prime}$. We conclude that $g^{2}=i d_{V}$, and that either $N=C$, or $N=C \rtimes\langle g\rangle$. The case $N=C$ is impossible because $C \not A$. Let $N=C \rtimes\langle g\rangle$. Then $\left(S_{i}^{-}\right)^{g}=S_{i}^{-}$(for both $i \in\{1,2\}$ ), hence $S_{i}$ is a union of orbits of $g$. As $g$ normalizes $C$ and fixes $0^{+}, g=\varphi_{r, 0, s}$. Recall that $\operatorname{ord}(g)=2$. If $r \neq 1$, then by Lemma 3.6, either $C$ is the unique cyclic subgroup of $N$, or $8 \mid n, r=n / 2+1$ and $s=0$ or $s=n / 2$. In the former case $C$ is characteristic in $N$, and since $N \unlhd A, C \unlhd A$, a contradiction. Therefore, we are left with the case that $r=1$ (and so $s=n / 2$ ), or $8 \mid n$, $r=n / 2+1$ and $s=0$ or $s=n / 2$. Then every orbit of $g$ is of length 1 or 2 , and if it is of length 2 , then is in the form $\left\{j^{\varepsilon},(j+m)^{\varepsilon}\right\}$ as we proved in Case 1 . Since $n_{i}>2$, we see that $S_{i}^{-}$must be fixed pointwise by $g$ for both $i \in\{1,2\}$. This, however, contradicts that $g$ was assumed to act non-trivially on $S^{-}$; and so $n_{1}=2$.

This means that $2 \mid n$, say $n=2 m$, and the group generated by the set $S_{1}-S_{1}=$ $\left\{x-y: x, y \in S_{1}\right\}$ is equal to $\{0, m\}$. Then we can write $S_{1}=\{s, s+m\}$. It can be proved as before that there exist $a \in \mathbb{Z}_{n}^{*}$ and $b \in \mathbb{Z}_{n}$ such that $a S_{2}+b=\{0, u\}$ for some divisor $u$ of $n$. Then, letting $v=a s+b$, we get $a S_{1}+b=\{v, v+m\}$. We finish the proof of this case by showing that the set $R=a S+b=\{0, u, v, v+m\}$ satisfies the conditions (a)-(c) of Theorem 3.1 .

(a): As $H\left(\mathbb{Z}_{n}, S\right)$ is connected, $H\left(\mathbb{Z}_{n}, R\right)$ is also connected. This implies that $\{u, v\}$ is a generating set of $\mathbb{Z}_{n}$.

(c): Since $S_{1}$ and $S_{2}$ are left fixed setwise by $A, \operatorname{Aut}\left(H\left(\mathbb{Z}_{n}, R\right)\right)_{0^{+}}$leaves the set $\left\{0^{-}, u^{-}\right\}$setwise fixed.

(b): If $u=1$, then $\operatorname{Aut}\left(H\left(\mathbb{Z}_{n},\{0, u\}\right)\right) \leq D_{4 n}$. But then $C \unlhd A$, which is a contradiction. We conclude that $1<u$, and by Lemma 4.4, $u \mid m$ also holds, i.e., $1<u<m$ and $u \mid m$, as required.

\section{Acknowledgements}

In this research both authors have been supported in part by "Javna agencija za raziskovalno dejavnost Republike Slovenije”, program no. P1-0285. The authors are much grateful to one of the anonymous referees, whose constructive comments helped to improve the quality of the presentation considerably. 


\section{References}

[1] L. Babai, Isomorphism problem for a class of point-symmetric structures, Acta Math. Acad. Sci. Hungar. 29 (1977), 329-336.

[2] M. Boben, T. Pisanski, and A. Žitnik, I-graphs and the corresponding configurations, J. Combin. Designs 13 (2005), 406-424.

[3] T. G. Boreham, I. Z. Bouwer, and R. W. Frucht, A useful family of bicubic graphs, Graphs Combin. (1973), 213-225.

[4] W. Bosma, J. Cannon, and C. Playoust, The Magma Algebra System I: The User Language, J. Symbolic Comput. 24 (1997), 235-265.

[5] J. D. Dixon and B. Mortimer, Permutation groups, Graduate Texts in Mathematics 163, Springer-Verlag, New York, 1996.

[6] M. Hladnik, D. Marušič, and T. Pisanski, Cyclic Haar graphs, Discrete Math. 244 (2002), 137152.

[7] W. Jin, W. Liu, A classification of nonabelian simple 3-BCI-groups, European J. Combin. 31 (2010), 1257-1264.

[8] I. Kovács, B. Kuzman, and A. Malnič, On non-normal arc transitive 4-valent dihedrants, Acta Math. Sinica (Engl. ser.) 26 (2010), 1485-1498.

[9] I. Kovács, B. Kuzman, A. Malnič, and S. Wilson, Characterization of edge-transitive 4-valent bicirculants, J. Graph Theory 69 (2012), 441-463.

[10] C. H. Li, On isomorphisms of finite Cayley graphs - a survey, Discrete Math. 256 (2002), 301-334.

[11] M. Muzychuk, On the isomorphism problem for cycic combinatorial objects, Discrete Math. 197/198 (1999), 589-606.

[12] M. Muzychuk, A solution of the isomorphism problem for circulant graphs, London Math. Soc. 88 (2004), 1-41.

[13] H. Qu, J. Yu, On isomorphism of Cayley digraphs on dihedral groups, Australian J. Combin. 15 (1997), 213-220.

[14] P. Spiga, On the Cayley isomorphism problem for a digraph with 24 vertices, Ars Math. Contemp. 1 (2008), 38-43.

[15] L. Sun, Isomorphisms of circulants with degree 2, J. Beijing Ins. Technol. 9 (1984), 42-46.

[16] D. Wiedemann, M. E. Zieve, Equivalence of sparse circulants: the bipartite Ádám problem, preprint arXiv:0706.1567v1 [math. CO] (2007).

[17] S. J. Xu, W. Jin, Q. Shi, and J. J. Li, The BCI-property of the Bi-Cayley graphs, J. Guangxi Norm. Univ.: Nat. Sci. Edition 26 (2008), 33-36. 